\title{
Ueber die geradlinigen Flächen fünften Grades.
}

\author{
( Von Herrn H. Schwarz.)
}

Die vorliegende Abhandlung ist eine weitere Ausführung einer Notiz über die geradlinigen Flächen fünften Grades, welche der Verfasser im Juni 1864 der philosophischen Facultät hiesiger Universität vorgelegt hat.

Da die geradlinigen Flächen dritten und vierten Grades schon von den Herren Cayley, Cremona und Salmon eingehend untersucht, worden sind, darf sich der Verfasser im Folgenden auf die Betrachtung der geradlinigen Flächen des fünften Grades beschränken, ohne auf diejenigen der niederen Grade einzugehen. -

Mit Ausnahme der erzeugenden Geraden gehören alle ebenen Curven, welche auf derselben irreduciblen geradlinigen Fläche liegen, und die Eigenschaft haben, dass durch jeden ihrer Punkte nur eine Erzeugende geht, in dem von Riemann aufgestellten Sinne zu derselben algebraischen Klasse.

Betrachtet man nämlịch irgend zwei einfache ebene Curven auf derselben irreduciblen geradlinigen Fläche, so wird eirem jeden Punkte der einen Curve durch die Erzeugenden der Fläche ein Punkt der anderen Curve auf eindeutige Weise zugeordnet, und es lassen sich daher die Coordinaten eines Punktes der einen Curve rational ausdrücken durch die Coordinaten des entsprechenden Punktes der anderen Curve und umgekehrt.

Aus diesem Grunde kann man die geradlinigen Flächen nach der algebraischen Klasse der auf ihnen liegenden ebenen Curven selbst in Klassen eintheilen. (S. d. Verf.: De superficiebus in planum explicabilibus primorum septem ordinum, Bd. 64, pag. 2 dieses Journals.)

Aus der Existenz einer einzigen einfachen geraden Leitlinie auf der Fläche, eines einfachen Kegelschnitts, einer einfachen Curve dritten Grades mit Doppelpunkt, oder überhaupt einer einfachen Curve mit der grössten Anzahl von Doppelpunkten kann daher der Schluss gezogen werden, dass die geradlinige Fläche zur Klasse $\varrho=0$ gehört.

Findet sich auf der Fläche eine einfache ebene Curve dritten Grades ohne Doppelpunkt, eine Curve vierten Grades mit zwei Doppelpunkten, überhaupt eine einfache ebene Curve $n^{\text {ten }}$ Grades mit $\frac{(n-1)(n-2)}{1.2}-1$ Doppelpunkten, so gehört die Fläche zur Klasse $\varrho=1$. 
Enthält die Fläche eine einfache ebene Curve vierten Grades mit nur einem Doppelpunkt, so gehört die Fläche zur Klasse $\varrho=2$. U. s. w.

Die Coordinaten eines beliebigen Punktes einer algebraischen Fläche lassen sich als rationale Functionen zweier variablen Parameter $s$ und $t$ und einer algebraischen Function $u$ derselben darstellen. Bei den geradlinigen Flächen ist es stets möglich, diese Parameter so zu wählen, dass die Ausdrücke der homogenen Coordinaten

$$
x: y: z: w
$$

eines beliebigen Punktes der Fläcbe ganze lineare Functionen des einen Parameters $s$ sind, während die algebraische Grösse $u$ nur von dem anderen Parameter $t$ abhängt.

Der andere Parameter $t$ kann dann so gewählt werden, dass die Coordinaten, wenn $\varrho=0$ ist, in Bezug auf denselben rationale ganze Functionen sind; wenn $\varrho=1$, rational ausgedrückt sind durch $t$ und eine Quadratwurzel aus einer ganzen Function dritten oder vierten Grades von $t$; wenn $\varrho=2$, rational ausgedrückt sind durch $t$ und eine Quadratwurzel aus einer ganzen Function fünften oder sechsten Grades von $t$. - Der Fall $\varrho>2$ führt auf höhere algebraische Irrationalitäten.

Diese Beziehungen gelten auch umgekehrt, so dass, wenn man für $x: y: z: w$ Ausdrücke setzt, die in Bezug auf $t$ und $u$ rational, in Bezug auf $s$ linear und ganz sind, während zwischen $t$ und $u$ eine irreducible algebraische Gleichung $f(t, u)=0$ besteht, man eine algebraische geradlinige Fläche erhält, welche im Allgemeinen zu derselben algebraischen Klasse gehört, zu welcher die Gleichung $f(t, u)=0$ gehört.

Zur geometrischen Construction der besonderen Fälle der geradlinigen Flächen fünften Grades werde ich mich meist•der Anschauung bedienen, die Flächen entstehen zu lassen als geometrischen Ort der geraden Verbindungslinien entsprechender Punkte zweier Curven, welche in der Weise auf einander bezogen sind, dass jedem Punkte der einen ein Punkt der anderen entspricht und umgekehrt.

Für die analytische Darstellung aber ist es mitunter vorzuziehen, aus den Ausdrücken $x: y: z: w$ durch Elimination von $s$ zwei in Bezug auf $x, y$, $z, w$ lineare homogene Gleichungen hergeleitet zu denken, in den Coefficienten rational in $t$ und $u$, so dass die Fläche der geometrische Ort der Durchschnittslinien der durch diese Gleichungen dargestellten Ebenen ist. 


\section{\$. 1.}

Allgemeine Untersuchung der verschiedenen möglichen geradlinigen Flächen fünften Grades.

Eine durch eine Erzeugende einer geradlinigen Fläche fünften Grades gelegte Ebene hat ausser der Erzeugenden mit der Fläche noch ein ebenes Gebilde vierten Grades gemein.

Von den vier Durchschnittspunkten der Geraden mit diesem Gebilde ist nur einer Berührungspunkt der Ebene und der Fläche; die anderen drei Durchschnittspunkte sind zugleich Doppelpunkte der Fläche, durch welche daher ausser der einen Erzeugenden noch je eine zweite Erzeugende hindurchgeht.

Jede Erzeugende der Fläche wird also im Allgemeinen von drei anderen Erzeugenden geschnitten, und es giebt daher unendlich viele Ebenen, welche durch zwei Erzeugende der Fläche hindurchgehen.

Die Betrachtung dieser Ebenen bietet den Ausgangspunkt für unsere Untersuchung.

Eine jede Ebene, welche durch zwei erzeugende Gerade einer geradlinigen Fläche fünften Grades hindurchgeht, schneidet ausser den beiden Geraden noch ein Gebilde dritten Grades aus. Dieses Gebilde kann irreducibel sein oder selbst wieder zerfallen.

Wenn überhaupt der Schnitt einer Ehene und einer irreduciblen geradlinigen Fläche zerfällt, so kann er nur in eine gewisse Anzahl Erzeugende und einen irreduciblen Theil von der Beschaffenheit zerfallen, dass durch jeden Punkt desselben mindestens eine Erzeugende geht, vorausgesetzt, dass die Fläche nicht eine Kegelfläche ist und der Schnitt durch dessen Mittelpunkt geführt wird.

Die Kegelflächen schliessen wir von unserer Betrachtung aus.

Im vorliegenden Falle kann der irreducible Theil sein eine einfache oder mehrfache Gerade, durch welche alle Erzeugenden der Fläche hindurchgehen, ein Kegelschnitt oder eine Curve dritten Grades.

A. Ist der irreducible Theil eine einfache Gerade, ein Kegelschnitt oder eine Curve dritten Grades mit einem Doppelpunkt, so gehört die geradlinige Fläche zur ersten Klasse $(\varphi=0)$.

Es ist übrigens zu bemerken, dass auf einer irreduciblen geradlinigen Fläche von höherem als dem vierten Grade nur ein einfacher Kegelschnitt liegen kann, weil durch zwei einfache Kegelschnitte, welche Punkt für Punkt 
eindeutig auf einander bezogen sind, stets eine geradlinige Fläche vierten Grades bestimmt wird.

$B$. Ist der irreducible Theil eine Curve dritten Grades ohne Doppelpunkt, so gehört die geradlinige Fläche zur zweiten Klasse $(\omega=1)$.

C. Eine besondere Betrachtung ist nun für den Fall anzustellen, dass auf der geradlinigen Fläche fünften Grades eine gerade Leitlinie vorhanden ist, welche eine mehrfache Linie der Fläche ist.

a. Ist die Gerade eine zweifache, so schneidet jede durch dieselbe gelegte Ebene aus der Fläche drei Erzeugende aus, weil der Schnitt dieser Ebene ausser der zweifachen Leitgeraden keinen irreduciblen Theil mehr enthalten kann.

Durch jeden Punkt der Doppelgeraden geht nun entweder nur eine von ihr selbst verschiedene Erzeugende, und dann gehört die Fläche zur ersten Klasse, oder es gehen deren durch jeden Punkt zwei hindurch.

Im letzteren Falle geht die Ebene dieser beiden Erzeugenden im Allgemeinen nicht durch die Doppelgerade hindurch.

Gesetzt nämlich, dies wäre der Fall, so enthielte jede durch die Doppelgerade gehende Ebene immer zwei Erzeugende, welche sich auf der Doppelgeraden schneiden, und eine dritte Erzeugende, welche nicht auch noch durch den Schnittpunkt der beiden anderen hindurchgehen kann, weil die Leitgerade nur eine zweifache ist.

Diese dritte Erzeugende schneidet also die Doppelgerade in einem anderen Punkte. Durch denselben muss noch eine zweite Erzeugende der Fläche gehen, die aber nicht mehr in der betrachteten Ebene liegen kann. Die Ebene der beiden durch den letztgenannten Punkt gehenden Erzeugenden enthält also die Doppelgerade nicht. Die Voraussetzung, dass die Ebene der zwei durch denselben Punkt der Doppelgeraden gehenden Erzeugenden die Doppelgerade stets enthalten könne, ist demnach für den Fall einer irreduciblen Fläche als nicht zulässig erwiesen.

Betrachten wir nun den Schnitt der Ebene, welche die zwei durch denselben Punkt der Doppelgeraden gehenden Erzeugenden enthält, so kann diese keine dritte Erzeugende mehr enthalten, weil diese auch durch die Leitgerade gehen müsste, welche der Voraussetzung zufolge nur eine doppelte Linie der Fläche ist. Diese Ebene schneidet daher aus der Fläche entweder eine irreducible Curve dritten Grades (s. A. und B.) oder eine dreifache Gerade aus.

Legt man im letzten Falle, in welchem die Fläche eine zweifache und 
eine dreifache Leitgerade hat, durch eine Erzeugende der Fläche eine Ebene, welche keine der beiden geraden Leitlinien enthält, so schneidet sie ausser der Erzeugenden aus der Fläche eine ebene Curve vierten Grades aus, welche in dem Punkte, in welchem die Ebene von der dreifachen Geraden geschnitten wird, einen Doppelpunkt besitzt.

Enthält nun die geradlinige Fläche keine doppelte Erzeugende, so hat die Curve vierten Grades keinen weiteren Doppelpunkt und gehört demnach zur dritten Klasse $(\varrho=2)$; enthält die Fläche aber eine oder zwei Doppelerzeugende, so gehört sie zur zweiten, beziehlich zur ersten Klasse, weil dann die Curve vierten Grades noch einen, beziehlich zwei Doppelpunkte erhält.

Jede geradlinige Fläche fünften Grades, welche eine doppelte Erzeugende enthält, die nicht zugleich eine Leitgerade ist, enthält unendlich viele ebene Curven dritten Grades, welche von dem Ebenenbüschel ausgeschnitten werden, das die Doppelgerade zur Axe hat.

b. Ist der von einer Ebene, welche zwei Erzeugende enthält, ausgeschnittene irreducible Theil eine dreifache Gerade, so können drei Fälle eintreten: durch jeden Punkt derselben gehen entweder nur eine, oder zwei, oder drei von der Leitlinie verschiedene Erzeugende hindurch.

Geht durch jeden Punkt der Leitlinie nur eine von derselben verschiedene Erzeugende, so gehört die Fläche zur ersten Klasse.

Gehen durch jeden Punkt derselben zwei von der Leitlinie verschiedene Erzeugende, und geht die Ebene derselben nicht durch die dreifache Gerade, so schneidet diese Ebene aus der Fläche entweder eine irreducible Curve dritten Grades aus, - für eine besondere Lage möglicherweise einen Kegelschnitt, - oder eine mehrfache, - in diesem Falle doppelte, gerade Leitlinie aus. Beide Fälle sind bereits erledigt.

Geht aber die Ebene der zwei Erzeugenden stets durch die dreifache Gerade hindurch, schneiden sich also die beiden von einer beliebigen durch die dreifache Gerade gelegten Ebene ausgeschnittenen Erzeugenden stets auf der dreifachen Geraden, so giebt es ausser dieser Geraden und möglicherweise vorhandenen Doppelerzeugenden keine mehrfache Linie auf der Fläche.

Legt man in diesem Falle durch eine Erzeugende eine Ebene, so schneidet diese noch eine Curve vierten Grades aus, welche an der Stelle, wo die Ebene von der dreifachen Geraden geschnitten wird, einen Doppelpunkt besitzt. Die Fläche gehört also, wenn sie nicht Doppelerzeugende enthält, in die dritte Klasse $(\varphi=2)$. 
Schwarz, über die geradlinigen Flächen fünften Grades.

Ist drittens die dreifache Gerade so beschaffen, dass durch jeden Punkt derselben drei von ihr verschiedene Erzeugende gehen, so muss eine Ebene existiren, welche zwei derselben enthält, ohne die dreifache Gerade zu enthalten; diese Fbene schneidet nun entweder eine irreducible Curve dritten Grades aus oder eine mehrfache (zweifache) Gerade, Fälle, die bereits klassificirt sind.

Endlich gehört hierher noch der Fall, dass der ausgeschnittene irreducible Theil eine dreifache Gerade ist, mit welcher aber die eine Erzeugende. stets zusammenfällt; die Fläche fünften Grades enthält dann also eine vierfache Gerade.

Jede Ebene, welche durch eine vierfache Gerade einer Fläche fünften Grades gelegt wird, schneidet aus der Fläche nur noch eine Gerade aus. Daher ist jede Fläche fünften Grades mit einer vierfachen Geraden eine geradlinige Fläche und gehört als solche zur ersten Klasse.

Wir fassen nun die Ergebnisse der Untersuchung dieses Paragraphen wie folgt zusammen:

Eine irreducible geradlinige Fläche füften Grades, welche keine Kegelfläche ist, ist entweder von der ersten, oder von der zweiten oder von der dritten (algebraischen) Klasse.

Die Flächen der ersten und zweiten Klasse enthalten, mit einer gleich anzugebenden Ausnahme, eine unendliche Schaar von Curven dritten Grades, und demnach ist eine solche Fläche geometrisch construirbar durch Vermittelung zweier Curven dritten Grades, die eindeutig auf einander bezogen werden.

Hiervon sind ausgenommen und es enthalten keine einzige irreducible Curve dritten Grades diejenigen Flächen der ersten Klasse, welche eine einfache gerade Leitlinie haben, durch deren jeden Punkt nur eine oon der Leitlinie verschiedene Erzeugende geht.

\section{2.}

Besondere Betrachtung der geradlinigen Flächen fünften Grades, welche zur ersten Riemannschen Klasse gehören.

Die Gleichungen der beiden Ebenen, durch deren Durchschnitt für jeden Werth des Parameters $t$ eine Erzeugende der geradlinigen Fläche bestimmt wird, seien

$$
\left.\begin{array}{l}
E=a t^{m}+b t^{m-1}+\cdots+p t+q=0 \\
E^{\prime}=a^{\prime} t^{n}+b^{\prime} t^{n-1}+\cdots+p^{\prime} t+q^{\prime}=0
\end{array}\right\} \quad m \geqq n,
$$


wobei $a, b, \ldots q, a^{\prime}, b^{\prime}, \ldots q^{\prime}$ homogene lineare Functionen der vier homogenen Coordinaten darstellen.

Die Elimination von $t$ ergiebt eine homogene Gleichung, welche in Bezug auf $a, b, \ldots q$ vom $n^{\text {ten }}$ Grade, in Bezug auf $a^{\prime}, b^{\prime}, \ldots q^{\prime}$ vom $m^{\text {ten }}$ Grade ist, stellt also eine Fläche vom $m+n^{\text {ten }}$ Grade dar.

Wenn die beiden Ausdrücke $E$ und $E^{\prime}$ für jeden Werth von $t$ nur eine Gerade, also für keinen Werth von $t$ eine ganze Ebene darstellen, so kann die Eliminationsgleichung keinen ausserwesentlichen Factor enthalten.

Stellen aber $E$ und $E^{\prime}$ für einen Werth $t=t_{0}$ dieselbe Ebene dar, so geht die Gleichung derselben als ausserwesentlicher Factor in die Eliminationsgleichung ein. In diesem Falle denken wir eine Constante $k$ so bestimmt, dass $E-k E^{\prime}$ für $t=t_{0}$ identisch verschwindet, was stets möglich ist. $E-k E^{\prime}$ ist durch $t-t_{0}$ theilbar, so dass $E-k E^{\prime}=\left(t-t_{0}\right) E^{\prime \prime}$. An die Stelle der Ebene $E=0$ setzen wir nun die Ebene $E^{\prime \prime}=0$. Hiermit fahren wir so lange fort, bis kein Werth von $t$ mehr übrig ist, für den beide Ausdrücke gleichzeitig eine ganze Ebene darstellen.

Die Möglichkeit, dass die Eliminationsgleichung, welche jetzt keinen ausserwesentlichen Factor mehr enthalten kann, eine Potenz sei, d. h. dass jede Erzeugende durch beide Gleichungen für mehr als einen Werth von $t$ dargestellt werde, schliessen wir hier aus, da wir wissen, dass die zu betrachtenden Flächen so dargestellt werden können, dass jeder einfachen Erzeugenden nur ein Werth des Parameters entspricht.

Soll also die Eliminationsgleichung irreducibel und vom fünften Grade sein, so ist $m+n$ gleich $5 \mathrm{zu}$ setzen. Es sind demnach nur die beiden Fälle zulässig:

$$
\begin{aligned}
& \text { (I.) } \quad\left\{\begin{aligned}
m=4 ; & E=a t^{4}+b t^{3}+c t^{2}+d t+e=0, \\
n=1 ; & E^{\prime}=p t+q=0 ;
\end{aligned}\right. \\
& \text { (II.) } \quad\left\{\begin{aligned}
m=3 ; & E=a t^{3}+b t^{2}+b^{\prime} t+a^{\prime}=0, \\
n=2 ; & E^{\prime}=p t^{2}+q t+r=0 .
\end{aligned}\right.
\end{aligned}
$$

A. Da der Grad einer geradlinigen Fläche durch reciproke Verwandlung nicht geändert wird, ist es erlaubt, von diesen Ebenengebilden gleich zu den entsprechenden Punktgebilden überzugehen. Wir erhalten also folgende Sätze:

I. Besteht zwischen den Punkten einer Geraden und den Punkten einer Curve vierten Grades, welche zu der rationalen Klasse gehört, ein eindeutiges 
gegenseitiges Entsprechen, so ist der geometrische Ort der Verbindungslinien entsprechender Punkte eine geradlinige Fläche fünften Grades.

II $^{a}$. Derselbe Satz gilt, wenn man gleichzeitig an die Stelle der Geraden einen Kegelschnitt und an die Stelle der Curve vierten Grades eine Curve dritten Grades setzt.

Ein solches Entsprechen kann geometrisch in allgemeinster Weise durch mannigfaltige Beziehungen, z. B. dadurch hergestellt werden, dass man sich den Kegelschnitt und die Raumcurve dritten Grades auf demselben Kegel zweiten Grades denkt und die Punkte beider durch die Seiten des Kegels eindeutig einander zuordnet.

Als Grenzfall ist besonders zu betrachten der Fall, in welchem der Kegelschnitt zu einer doppelten Geraden wird.

$\mathrm{II}^{b}$. Ordnet man den Punkten einer Curve dritten Grades, welche zur rationalen Klasse gehört, in der Weise die Punkte einer Geraden zu, dass jedem Punkte der ersteren ein Puntet der letzteren, jedem Punkte der letzteren aber zwei Punkte der ersteren entsprechen, so ist der geometrische Ort der Verbindungslinien entsprechender Punkte eine geradlinige Fläche füften Grades.

Setzt man im zweiten Falle an die Stelle der Ebene $E^{\prime}$ eine Ebene $E+k\left(t-t_{0}\right) E^{\prime}=0$, so gelangt man, dem Vorigen entsprechend, zu dem Satze:

II. Man erhält eine geradlinige Fläche fünften Grades, wenn man die Punkte zweier Curven dritten Grades, welche zur rationalen Klasse gehören, eindeutig auf einander bezieht, einen Punkt der einen mit dem ihm entsprechenden Punkte der anderen zusammenfallen lässt und die entsprechenden Punkte durch gerade Linien mit einander verbindet.

Aus den angestellten Betrachtungen geht hervor, dass jede geradlinige Fläche füften Grades, welche zu der rationalen Klasse gehört, durch eine der angegebenen Constructionen I. und II., oder I., II ${ }^{a}$., II ${ }^{b}$. erhalten werden kann.

$B$. Wir wollen uns nun mit der Doppelcurve der betrachteten Flächen etwas beschäftigen und darauf $(C)$ einige Fälle, in denen sie reducibel wird, näher ins Auge fassen, ohne uns jedoch hierbei ein Erschöpfen des Stoffes zum Ziele zu setzen. Die einzelnen Arten der geradlinigen Flächen fünften Grades, die sich durch die Betrachtung der Doppelcurve ergeben, bezeichnen wir in jeder Klasse der Reihe nach durch beigesetzte römische Ziffern.

In dem ersten Falle ( $s$. die Gleichungen auf der vorigen Seite) ist die Gerade $p=0, q=0$ eine vierfache Gerade der Fläche. - (I.) 
In dem zweiten Falle ist die Gleichung der Fläche

$$
\left|\begin{array}{lllll}
a & b & b^{\prime} & a^{\prime} & 0 \\
0 & a & b & b^{\prime} & a^{\prime} \\
p & q & r & 0 & 0 \\
0 & p & q & r & 0 \\
0 & 0 & p & q & r
\end{array}\right|=0 .
$$

Durch theilweise Elimination erhält man die Gleichungen

$$
\begin{aligned}
(a q-b p) t^{2}+\left(a r-b^{\prime} p\right) t-a^{\prime} p & =0, \\
a r t^{2}+\left(b r-a^{\prime} p\right) t+b^{\prime} r-a^{\prime} q & =0 ;
\end{aligned}
$$

es lässt sich also die Gleichung der Fläche auch in folgende Form setzen

$$
\left|\begin{array}{ccc}
a q-b p & a r-b^{\prime} p & -a^{\prime} p \\
p & q & r \\
a r & b r-a^{\prime} p & b^{\prime} r-a^{\prime} q
\end{array}\right|=0 .
$$

Indem man fortfährt, zwischen den beiden soeben erhaltenen Gleichungen, welche in Bezug auf $t$ vom zweiten Grade sind, und der Gleichung $E^{\prime}=0$ zu eliminiren, erhält man folgende Gleichungen

wenn man die Ausdrücke

$$
\begin{aligned}
& \varphi_{1} t+\varphi_{2}=0 \\
& \varphi_{2} t+\varphi_{3}=0, \\
& \varphi_{3} t+\varphi_{4}=0,
\end{aligned}
$$

$$
\begin{gathered}
(a q-b p) q-\left(a r-b^{\prime} p\right) p, \\
(a q-b p) r+a^{\prime} p^{2}=a q r-\left(b r-a^{\prime} p\right) p, \\
\left(a r-b^{\prime} p\right) r+a^{\prime} p q=a r^{2}-\left(b^{\prime} r-a^{\prime} q\right) p \\
\left(b r-a^{\prime} p\right) r-\left(b^{\prime} r-a^{\prime} q\right) q
\end{gathered}
$$

der Reihe nach mit $\varphi_{1}, \varphi_{2}, \varphi_{3}, \varphi_{4}$ bezeichnet. Die Gleichung der Fläche erscheint nun unter den Formen

$$
\begin{aligned}
\varphi_{2}^{2}-\varphi_{1} \varphi_{3} & =0 \\
\varphi_{1} \varphi_{4}-\varphi_{2} \varphi_{3} & =0 \\
\varphi_{3}^{2}-\varphi_{2} \varphi_{4} & =0
\end{aligned}
$$

in welchen sie mit den ausserwesentlichen Factoren $p=0, q=0, r=0$ behaftet ist.

Aus den identischen Gleichungen

$$
\begin{aligned}
& r \varphi_{1}-q \varphi_{2}+p \varphi_{3}=0 \\
& r \varphi_{2}-q \varphi_{3}+p \varphi_{4}=0
\end{aligned}
$$


geht hervor, dass die Flächen $\varphi_{1}=0, \varphi_{2}=0, \varphi_{3}=0, \varphi_{4}=0$ durch dieselbe Curve hindurchgehen, wenn diejenigen reduciblen Theile ihrer Durchschnitte ausgeschlossen werden, die in den Ebenen $p=0, q=0, r=0$ liegen; ferner geht aus denselben, in Verbindung mit den Gleichungen der Fläche, hervor, dass diese allen vier Flächen gemeinschaftliche Curve eine Doppelcurve der Fläche ist.

Die Flächen $\varphi_{2}=0$ und $\varphi_{3}=0$ haben drei gerade Linien gemeinschaftlich:

$$
\begin{aligned}
p & =0, & r & =0, \\
p & =0, & a & =0, \\
r & =0, & a^{\prime} & =0,
\end{aligned}
$$

also ist die Curve, welche sie ausserdem gemeinschaftlich haben, vom sechsten Grade; dieselbe hat im Punkte $p=0, q=0, r=0$ einen dreifachen Punkt, weil durch diesen Punkt, der für die.Flächen $\varphi_{2}=0$ und $\varphi_{3}=0$ ein Doppelpunkt, also für deren Durchschnittslinie ein vierfacher Punkt ist, nur eine der drei den beiden Flächen gemeinschaftlichen Geraden geht.

Die Fläche $\varphi_{2}^{2}-\varphi_{1} \varphi_{3}=0$ wird umhüllt von der Flächenschaar

$$
\varphi_{1} t^{2}+2 \varphi_{2} t+\varphi_{3}=0
$$

jede dieser Flächen dritten Grades schneidet die geradlinige Fläche fünften Grades in der Doppelcurve sechsten Grades, berührt dieselbe längs einer Erzeugenden und geht ausserdem noch durch eine Erzeugende der Fläche hindurch. Dasselhe gilt für die Flächenschaar

$$
\varphi_{2} t^{2}+2 \varphi_{3} t+\varphi_{4}=0 .
$$

Jede Erzeugende der Fläche schneidet dreimal die Doppelcurve, also kann diese nicht auf einer Fläche zweiten Grades liegen, weil sonst jede Erzeugende der Fläche zugleich Erzeugende der Fläche zweiten Grades sein müsste. - Durch jeden Punkt der Curve gehen zwei Erzeugende, von denen jede die Curve noch zweimal schneidet; der Kegel fünften Grades, welcher die Doppelcurve zur Leitlinie und einen Punkt derselben zum Mittelpunkt hat, enthält also zwei doppelte und eine dreifache Erzeugende wegen des dreifachen Punktes; die Curve sechsten Grades gehört also im Allgemeinen zur Klasse $\varrho=1$.

Da auch eine vierfache Gerade für eine Doppelcurve sechsten Grades zählt, so haben wir den Satz:

Alle geradlinigen Flächen fünften Grades und erster Klasse haben eine 
Doppelcurve vom sechsten Grade, welche nothwendig einen dreifachen Punkt besitzt. - (II.)

Derselbe lässt sich wie folgt umkehren:

Jede irreducible Fläche füften Grades mit einer Doppelcurve sechsten Grades ist eine geradlinige Fläche.

Die Richtigkeit dieses Satzes ist evident, wenn die Doppelcurve sechsten Grades eine irreducible ist, denn in diesem Falle giebt es, vorausgesetzt, dass die Doppelcurve nicht einen vierfachen Punkt hat, unendlich viele gerade Linien, welche die Doppelcurve in drei distincten Punkter schneiden, mit der Fläche sechs Punkte gemein haben und daher ganz auf der Fläche liegen müssen.

Der Kegel nämlich, für welchen eine Raumcurve Leitlinie ist, und dessen Mittelpunkt ein Punkt der Curve ist, hat für alle Raumcurven von höherem als dem vierten Grade Doppelkanten. Unter diesen sind, wenn der Punkt auf der Curve nicht in singulärer Lage gewählt wird, und die Curve, ist sie vom fünften Grade, nicht etwa einen dreifachen Punkt, ist sie vom sechsten Grade, nicht etwa einen vierfachen Punkt, ist sie vom $n^{\text {ten }}$ Grade, nicht einen $(n-2)$ fachen Punkt besitzt, jedesmal solche enthalten, welche die Curve in drei distincten Punkten schneiden.

Eine Doppelcurve sechsten Grades mit einem vierfachen Punkte kann aber eine irreducible Fläche fünften Grades aus dem Grunde nicht haben, weil der vierfache Punkt der Doppelcurve zugleich ein vierfacher Punkt der Fläche und der Kegel zweiten Grades, auf welchem die Curve liegt, reducibler Theil der Fläche sein würde.

Diese Schlussweise kann nicht ohne Weiteres auf alle Fälle ausgedehnt werden, in denen die Doppelcurve sechsten Grades in Curven niederer Grade zerfällt; eine genauere Untersuchung zeigt aber, dass der obige Satz auch in diesen Fällen noch richtig bleibt.

C. Die Doppelcurve sechsten Grades der geradlinigen Flächen fünften Grades und erster Klasse kann nun auf mannigfache Weise zerfallen.

Einige der hierbei möglichen Fälle wollen wir hier näher betrachten.

Es sei zunächst auf der Fläche eine dreifache Leitgerade vorhanden.

Sind $p_{1}=0$ und $q_{1}=0$ die Gleichungen zweier durch die dreifache Leitgerade gehenden Ebenen, so lässt sich jede andere durch dieselbe gehende Ebene durch die Gleichung $p_{1} \lambda_{1}+q_{1}=0$ darstellen.

Eine solche Ebene schneidet aus der Fläche zwei Erzeugende aus, Journal für Mathematik Bd. LXVII. Heft 1. 
die von der Leitgeraden im Allgemeinen verschieden sind; zu jedem Werthe von $\lambda_{1}$ gehören also zwei Werthe von $t_{1}$; zu jeder Erzeugenden, also auch zu jedem Werthe von $t_{1}$ gehört aber nur eine Ebene, die durch die Leitgerade geht, also auch nur ein Werth von $\lambda_{1}$.

Hieraus folgt, dass $\lambda_{1}$ eine rationale Function zweiten Grades von $t_{1}$ ist. Die zwischen $\lambda_{1}$ und $t_{1}$ bestehende Gleichung kann man durch lineare Substitution beider Variabeln auf die Form

$$
\lambda=t^{2}
$$

bringen. Denkt man sich dies ausgeführt, so erhält man als allgemeine Gleichungen einer geradlinigen Fläche fünften Grades mit einer dreifachen Leitgeraden

$$
\begin{gathered}
p t^{2}+q=0 \\
a t^{3}+b t^{2}+b^{\prime} t+a^{\prime}=0 ; \\
\left(a q-b^{\prime} p\right) t+\left(b q-a^{\prime} p\right)=0 \\
\left(a q-b^{\prime} p\right)^{2} q+\left(a^{\prime} p-b q\right)^{2} p=0 .
\end{gathered}
$$

Die beiden Ebenen $p=0$ und $q=0$ berühren die Fläche jede längs einer Geraden.

[Gehen durch jeden Punkt der dreifachen Geraden nicht drei, sondern nur zwei oder nur eine von derselben verschiedene Erzeugende, so ist die dreifache Gerade eine besondere Lage der Erzeugenden oder eine Doppelerzeugende der Fläche, und die Gleichung $a t^{3}+b t^{2}+b^{\prime} t+a^{\prime}=0$ muss dann für einen oder für zwei Werthe von $t$ eine durch die dreifache Gerade gehende Ebene darstellen, oder die Form haben:

$$
\begin{gathered}
\left(a t^{2}+b t+c\right)\left(t-t_{0}\right)+\alpha p+\beta q=0 \\
\left.(a t+b)\left(t-t_{0}\right)\left(t-t_{1}\right)+(\alpha p+\beta q) t+\alpha_{1} p+\beta_{1} q=0 .\right]
\end{gathered}
$$

Ausser der dreifachen Geraden $p=0, q=0$ enthält die Fläche als Doppelcurve die Raumcurve dritten Grades, in welcher sich die Flächen

$$
a q-b^{\prime} p=0, \quad b q-a^{\prime} p=0, \quad a a^{\prime}-b b^{\prime}=0
$$

schneiden; dieselbe hat mit der dreifachen Geraden zwei Punkte gemein. - (III.)

Diese Curve dritten Grades kann nun in einen Kegelschnitt und eine Gerade oder in drei Gerade zerfallen.

Damit dieselbe in einen Kegelschnitt und eine Gerade zerfalle, ist nothwendig und hinreichend, dass die beiden Flächen $a q-b^{\prime} p=0$ und $b q-a^{\prime} p=0$ ausser der Geraden $p=0, q=0$ noch eine Gerade der anderen Schaar ge- 
mein haben; wird diese durch die Ebene $p \lambda_{0}+q=0$ ausgeschnitten, so hat man die identische Gleichung

$$
a \lambda_{0}+b^{\prime}=\varkappa_{0}\left(b \lambda_{0}+a^{\prime}\right)+\mu_{0}\left(p \lambda_{0}+q\right) .
$$

Unter dieser Voraussetzung ist, ebenfalls identisch,

$$
a q-b^{\prime} p=\varkappa_{0}\left(b q-a^{\prime} p\right)+\left(a-\varkappa_{0} b-\mu_{0} p\right)\left(p \lambda_{0}+q\right),
$$

und es liegt der beiden Flächen gemeinschaftliche Kegelschnitt in der Ebene $a-\varkappa_{0} b-\mu_{0} p=s=0$.

Wir haben dann als Gleichung der Fläche

$$
\left[s\left(p \lambda_{1}+q\right)+\varkappa_{10}\left(b q-a^{\prime} p\right)\right]^{2} q+\left(b q-a^{\prime} p\right)^{2} p=0 .
$$

Die Gerade $p \lambda_{0}+q=0, a^{\prime}+b \lambda_{0}=0$ ist in diesem Falle eine doppelte Erzeugende der Fläche.

Haben beide Flächen zweiten Grades $a q-b^{\prime} p=0$ und $b q-a^{\prime} p=0$ vier Gerade gemein, zwei von jeder Schaar, so ist diejenige Gerade, welche mit $p=0, q=0 \mathrm{zu}$ derselben Schaar gehört, eine zweifache Leitlinie, während die beiden anderen Doppelerzeugende der Fläche sind.

Wir erhalten die allgemeine Gleichung dieser Fläche aus der vorigen, wenn wir die Bedingung hinzufügen, dass die Ebene $s=0$ durch zwei Erzeugende der Fläche $b q-a^{\prime} p=0$ hindurchgehen soll.

Wird die eine derselben ausgeschnitten durch die Ebenen $p \lambda_{1}+q=0$, $a^{\prime}+b \lambda_{1}=0$, so hat die Gleichung der Ebene $s$ die Form

$$
s=\alpha\left(p \lambda_{1}+q\right)+\beta\left(a^{\prime}+b \lambda_{1}\right),
$$

und diese Ebene schneidet die Fläche noch in der Geraden

$$
s=0, \quad \alpha\left(p \lambda_{0}+q\right)+\beta\left(a^{\prime}+b \lambda_{0}\right)=r=0 .
$$

Dann ist identisch

$$
b q-a^{\prime} p=\frac{1}{\beta\left(\lambda_{1}-\lambda_{0}\right)}\left[s\left(p \lambda_{0}+q\right)-r\left(p \lambda_{1}+q\right)\right] .
$$

Schreibt man nun $p^{\prime}$ für $p \lambda_{0}+q, q^{\prime}$ für $p \lambda_{1}+q$, so erhält die allgemeine Gleichung der Fläche die Form:

$$
\left(\mu p^{\prime} s-v q^{\prime} r\right)^{2} q+\left(\mu^{\prime} p^{\prime} s-\nu^{\prime} q^{\prime} r\right)^{2} p=0
$$

worin $\mu, \nu, \mu^{\prime}, \nu^{\prime}$ willkürliche Constanten bezeichnen; $p=0, q=0$ ist die dreifache, $r=0, s=0$ die zweifache gerade Leitlinie, $p^{\prime}=0, r=0$ und $q^{\prime}=0$, $s=0$ sind Doppelerzeugende der Fläche. -- (V.)

Die beiden Flächen zweiten Grades können sich auch längs der Geraden $p=0, q=0$ berühren, dann ist in dem Ausdrucke für $s$ die Constante 
$\beta$ gleich Null zu setzen, und wir erhalten als allgemeine Form der Gleichung der Fläche

$$
\left(\varkappa_{0}\left(b q-a^{\prime} p\right)+\alpha p^{\prime} q^{\prime}\right)^{2} q+\left(b q-a^{\prime} p\right)^{2} p=0 .
$$

Dann enthält die Fläche neben der dreifachen geraden Leitlinie eine überall unendlich nahe zweifache gerade Leitlinie und ausserdem zwei in den Ebenen $p^{\prime}=0$ und $q^{\prime}=0$ liegende doppelte Erzeugende. Das Hyperboloid $b q-a^{\prime} p=0$ berührt die Fläche längs der Geraden $p=0, q=0$ und schneidet diese als fünffache Gerade aus.

Entwickelt man die obige Gleichung, so wird für jeden Punkt in der Nähe der Geraden $p=0, q=0$ nur das Glied

$$
\left(p+x_{0}^{2} q\right)\left(b q-a^{\prime} p\right)^{2}
$$

unendlich klein von der dritten Ordnung. Die Ebene $p+x_{0}^{2} q=0$ ist eine Tangentialebene der Fläche längs dieser Geraden; dieselbe schneidet aus der Fläche diese Gerade vierfach aus.

Die beiden anderen Tangentialebenen der Fläche in jedem Punkte dieser Geraden fallen jedesmal mit der Tangentialebene der Fläche $b q-a^{\prime} p=0$ zusammen.

Man kann daher sagen, dass die betrachtete Fläche längs der Geraden $p=0, q=0$ des Hyperboloids $b q-a^{\prime} p=0$ sich selbst berühre.

Jede durch die Gerade $p=0, q=0$ gelegte Ebene schneidet aus der Fläche zwei sich auf der Leitgeraden schneidende Erzeugende aus. Denkt man sich einen Theil der Fläche durch stetige Veränderung der einen dieser Geraden, einen zweiten durch stetige Veränderung der zweiten Geraden erzeugt, so berühren sich beide Theile, und es rechtfertigt sich die gebrauchte Bezeichnung Selbstberïhrung für diese Singularität der Fläche. -

Die hier betrachtete Singularität ist dieselbe, welche Herr Cayley in seinem Second Memoir on Skew Surfaces otherwise Scrolls, Phil. Trans. vol. 154, (1865) pp. $559-577$ als line twofold bezeichnet.

Auf einen allgemeineren Fall als den betrachteten, der sich von ihm durch Wegfall der beiden Doppelerzeugenden unterscheidet, werden wir in \$. 4 zurückkommen.

Wir betrachten nun den Fall, in welchem die geradlinige Fläche fünften Grades eine doppelte Leitgerade hat.

Durch eine Ueberlegung, welche der im vorigen Falle der dreifachen Leitgeraden durchgeführten ganz analog ist, zeigt man, dass man aus dem 
allgemeinen Falle den vorliegenden erhält, wenn man festsetzt, dass die Ebenen $a=0, b=0, b^{\prime}=0, a^{\prime}=0$ durch dieselbe Gerade gehen.

Die Doppelcurve, welche die Fläche ausser der doppelten Leitgeraden hat, ist vom fünften Grade; dieselbe hat, ebenso wie im allgemeinen Falle die Doppelcurve sechsten Grades, einen dreifachen Punkt und liegt daher auf einem Kegel zweiten Grades, dessen Mittelpunkt dieser dreifache Punkt ist. In Folge dessen gehört sie zur rationalen Klasse und hat demnach ausser dem dreifachen Punkte noch drei scheinbare Doppelpunkte. Mit der doppelten Leitgeraden hat dieselbe zwei Punkte gemein. Umgekehrt ist jede Raumcurve fünften Grades mit den genannten Eigenschaften im Verein mit einer dieselbe zweimal schneidenden Geraden Doppelcurve einer geradlinigen Fläche fünften Grades. - (VI.)

[Von jedem Punkte der Geraden gehen nämlich zwei von der Geraden verschiedene die Curve fünften Grades zweimal schneidende Strahlen aus, und in jeder durch die Leitgerade gelegten Ebene liegen drei derselben.]

Die Curve fünften Grades kann nun dadurch zerfallen, dass eine doppelte Erzeugende auftritt. Diese Doppelgerade muss, weil sie auf dem Kegel zweiten Grades liegt, durch den dreifachen Punkt hindurchgehen; der Rest der Doppelcurve ist eine Curve vierten Grades mit einem Doppelpunkt, welche von der doppelten Leitgeraden in einem Punkte geschnitten wird. Umgekehrt ist auch eine Raumcurve vierten Grades mit einem Doppelpunkte im Verein mit einer Geraden, von der sie einmal geschnitten wird, Doppelcurve einer geradlinigen Fläche fünften Grades, welche ausserdem eine doppelte Erzeugende enthält. - (VII.)

Wir nehmen nun an, die Doppelcurve sechsten Grades zerfalle, ohne dass sich Leitgerade unter den Theilen befinden.

In zwei Curven dritten Grades kann die Doppelcurve sechsten Grades aus dem Grunde nicht zerfallen, weil die eine derselben von jeder Erzeugenden zweimal geschnitten werden müsste. Diese Bestimmung würde die andere Raumcurve einfach lassen.

Wir nehmen also an, die geradlinige Fläche habe einen doppelten Kegelschnitt; der Rest der Doppelcurve ist eine Curve vierten Grades und zwar mit einem Doppelpunkte, durch welchen der doppelte Kegelschnitt hindurchgeht. Ausserdem hat der Kegelschnitt noch zwei Punkte mit der Raumcurve vierten Grades gemein. - (VIII.)

Dies folgt daraus, dass die Doppelcurve einen dreifachen Punkt haben 
muss und daraus, dass die Ebene des doppelten Kegelschnitts, welche aus der Fläche nur noch eine Erzeugende ausschneidet, keinen mehrfachen Punkt enthalten kann, der nicht auf dem Kegelschnitt selbst liegt.

Die genannten Bedingungen sind auch dafür hinreichend, dass ein Kegelschnitt mit einer Raumcurve vierten Grades mit Doppelpunkt Doppelcurve einer geradlinigen Fläche fünften Grades sei.

Da wir vorausgesetzt hatten, dass die Fläche keine mehrfache gerade Leitlinie enthalte, enthält sie entweder eine einfache gerade Leitlinie oder einen einfachen Leitkegelschnitt.

Im ersteren Falle lassen sich die Gleichungen der Erzeugenden, wenn wir zu den reciproken Gebilden übergehen, auf die Form

$$
\begin{aligned}
a t^{4}+b t^{2}+c & =0 \\
p t+q & =0
\end{aligned}
$$

bringen.

Betrachtet man nun die einfache Leitgerade und die Doppelcurve vierten Grades als Leitlinien für einen Strahl, welcher die Leitgerade einmal und die Curve vierten Grades zweimal schneidet, so überzeugt man sich, dass ausser der geradlinigen Fläche fünften Grades noch eine geradlinige Fläche bestimmt wird, da die Curve vierten Grades zwei scheinbare Doppelpunkte hat und demnach von jedem Punkte der Leitgeraden zwei Strahlen ausgehen.

Eine Gerade und eine Curve vierten Grades mit zwei scheinbaren Doppelpunkten bestimmen auf diese Weise im Allgemeinen eine geradlinige Fläche achten Grades; denn jede durch die Gerade gelegte Ebene schneidet vier Doppelpunkte aus, entsprechend sechs Geraden, und die Gerade selbst ist eine doppelte.

Liegen auf einer geradlinigen Fläche fünften Grades eine einfache Leitgerade und eine Doppelcurve vierten Grades, so geht durch diese beiden Linien noch eine geradlinige Fläche dritten Grades, von der jede Erzeugende die Leitgerade einmal und die Doppelcurve vierten Grades zweimal schneidet.

Wir erhalten auch umgekehrt den Satz:

Die einfache Leitgerade einer geradlinigen Fläche dritten Grades bestimmt mit jeder Curve vierten Grades mit zwei scheinbaren Doppelpunkten, die auf der Fläche liegt, ausser der geradlinigen Fläche dritten Grades im Allgemeinen noch eine geradlinige Fläche füften Grades mit doppeltem Kegelschnitt.

Man kann bekanntlich auf jeder geradlinigen Fläche dritten Grades beliebig viele solche Curven vierten Grades erhalten, welche alle einen Dop- 
pelpunkt haben, indem man durch einen Kegelschnitt der Fläche, oder durch zwei auf der Doppelgeraden sich schneidende Erzeugende eine Fläche zweiten Grades legt; diese schneidet eine Curve der verlangten Beschaffenheit aus.

Umgekehrt kann man durch jede Raumcurve vierten Grades mit einem Doppelpunkt beliebig viele geradlinige Flächen dritten Grades legen. Durch den Doppelpunkt ziehe man eine Gerade, so ist der geometrische Ort der Strahlen, welche die Gerade einmal und die Curve zweimal schneiden, eine geradlinige Fläche dritten Grades.

Ganz analog kann man verfahren, indem man zwei Kegelschnitte, die sich in zwei Punkten schneiden, an die Stelle der Curve vierten Grades setzt.

Die einfache Leitgerade der geradlinigen Fläche dritten Grades, die man erhält, bestimmt mit den beiden Kegelschnitten ausser dieser Fläche dritten Grades noch eine geradlinige Fläche fünften Grades, welche neben diesen beiden Doppelkegelschnitten noch einen dritten Doppelkegelschnitt besitzt. - (IX.)

Wir gehen nun zur Betrachtung des anderen Falles über, in welchem die geradlinige Fläche einen einfachen Leitkegelschnitt und einen Doppelkegelschnitt besitzt. Drücken wir die Coordinaten beider Kegelschnitte rational durch je einen Parameter, $t$ und $s$ aus, so lassen sich dieselben stets so wählen, dass die zwischen ihnen bestehende Gleichung die Form $s=t^{2}$ hat.

Gehen wir zu den reciproken Gebilden über, so erhalten wir als Gleichungen der Erzeugenden

$$
\begin{aligned}
& a t^{4}+b t^{2}+c=0, \\
& p t^{2}+q t+r=0 ;
\end{aligned}
$$

die Ebenen, welche durch diese Gleichungen dargestellt werden, umhüllen die Kegel $b^{2}-4 a c=0$ und $q^{2}-4 p r=0$.

Damit durch die beiden Gleichungen eine geradlinige Fläche fünften Grades bestimmt werde, ist erforderlich, dass für einen bestimmten Werth $t=t_{0}$ die beiden Kegel eine gemeinsame Tangentialebene haben, - oder dass ein Punkt des einen Kegelschnitts mit seinem entsprechenden Punkte im anderen Kegelschnitte zusammenfalle. Wir setzen also voraus, dass identisch die Gleichung stattfinde

$$
a t_{0}^{4}+b t_{0}^{2}+c=p t_{0}^{2}+q t_{0}+r .
$$

Dann erhält man als gleichbedeutend mit den obigen Gleichungen die folgenden:

$$
\begin{aligned}
& E=\left[a\left(t^{2}+t_{0}^{2}\right)+b-p\right]\left(t+t_{0}\right)-q=0, \\
& E^{\prime}=p t^{2}+q t+r=0, \\
& a t^{4}+b t^{2}+c=E\left(t-t_{0}\right)+E^{\prime} .
\end{aligned}
$$


Betrachtet man nun den Durchschnitt der Fläche mit der Ebene $q=0$, so ergiebt sich

$$
\begin{aligned}
a t^{2}+a t_{0}^{2}+b-p & =0, \\
p t^{2}+r & =0 ;
\end{aligned}
$$

d. h. die Erzeugende, welche dem Werthe $t=t_{1}$ entsprichi, geht durch denselben Punkt der Ebene $q=0$, durch welchen die dem Werthe $t=-t_{1}$ entsprechende Erzeugende geht; der von der Ebene $q=0$ ausgeschnittene Kegelschnitt

$$
q=0, \quad p\left(a t_{0}^{2}+b-p\right)-a r=0,
$$

oder, wenn $t_{0}$ nicht gleich Null,

$$
\begin{aligned}
& a t_{0}^{2}+b-p=\frac{q t_{0}+r-c}{t_{0}^{2}}, \\
& q=0, \quad p r-p c-a r t_{0}^{2}=0,
\end{aligned}
$$

ist also ein Doppelkegelschnitt der Fläche.

Weil nun diese Fläche ebenso wie ihre reciproke einen Doppelkegelschnitt enthält und ebenso allgemein ist, ist es nicht nöthig zu der reciproken zurückzukehren; wir bleiben also gleich bei dieser stehen.

Die obige Formel zeigt, dass die Ebene $q=0$ die Erzeugende ausschneidet, welche sich für $t+t_{0}=0, t=-t_{0}$ ergiebt:

$$
\begin{array}{ll}
q=0, & p t_{0}^{2}-q t_{0}+r=0 \\
q=0, & p t_{0}^{2}+r=0
\end{array}
$$

Die Doppelcurve vierten Grades ergiebt sich als Durchschnitt der beiden Kegel

$$
\begin{aligned}
& p^{2}-a\left(p t_{0}^{2}-q t_{0}+r\right)=0, \\
& r^{2}-c\left(p t_{0}^{2}-q t_{0}+r\right)=0,
\end{aligned}
$$

deren gemeinschaftliche Tangentialebene $p t_{0}^{2}-q t_{1}+r=0$ ist, welche, wie gezeigt, die von der Ebene des doppelten Kegelschnitts ausgeschnittene Erzeugende enthält. Weil die beiden Kegel eine gemeinschaftliche Tangentialebene haben, so folgt, dass ihr Durchschnitt wirklich einen Doppelpunkt hat, wie wir schon vorher geschlossen.

Dass der Doppelkegelschnitt

$$
q=0, \quad p r-p c-a r t_{0}^{2}=0
$$

die Doppelcurve vierten Grades ausser in dem Doppelpunkte derselben $p=0$, $q=0, r=0$ noch in zwei ferneren Punkten schneidet, zeigt man analytisch wie folgt.

Setzt man in den Gleichungen der beiden Kegel $q=0$, so ist 


$$
\begin{aligned}
& p\left(p-a t_{0}^{2}\right)-a r=0 ; \quad \frac{p}{r}=\frac{a}{p-a t_{0}^{2}} \\
& r(r-c)-c p t_{0}^{2}=0 ; \quad \frac{p}{r}=\frac{r-c}{c t_{0}^{2}}
\end{aligned}
$$

Hieraus

$$
\frac{a}{p-a t_{0}^{2}}=\frac{r-c}{c t_{0}^{2}} ; \quad p r-p c-a r t_{0}^{2}=0 .
$$

Der Fall $t_{0}=0$, auf welchen wir uns den Fall $t_{0}=\infty$ zurückgeführt denken, erheischt eine besondere Betrachtung, indem dann die Gleichung des zweiten Kegels identisch erfüllt ist. Sełzt man jedoch für $c$ seinen Werth

$$
c=r+q t_{0}+(p-b) t_{0}^{2}-a t_{0}^{4},
$$

so erhält die Gleichung des Kegels den identischen Factor $t_{0}^{2}$; lässt man diesen fort, so ergiebt sich eine Gleichung, welche auch für $t_{0}=0$ einen bestimmten Sinn behält und dann in $q^{2}-2 r p+r b=0$ übergeht.

Aus dem zweiten Kegel ergiebt sich für $q=0, b-2 p=0$. Diese Ebene schneidet die Ebene $\boldsymbol{q}=\mathbf{0}$ in einer Geraden, deren Durchschnittspunkte mit dem Kegel $p^{2}$-ar auf dem Kegelschnitte $q=0, p(b-p)-a r=0$ liegen.

Es entsteht nun die Frage, welche Ebene schneidet aus der Fläche den einfachen Kegelschnitt aus?

Der dreifache Punkt der betrachteten geradlinigen Fläche ist ein Punkt des doppelten Kegelschnitts, und zwar liegt derselbe auf der von der Ebene des doppelten Kegelschnitts aus der Fläche ausgeschnittenen Geraden; also ist die dreifache Tangentialebene der Fläche eine Tangentialebene des umschriebenen doppelt berührenden Kegels $b^{2}-4 a c=0$ und enthält die durch dessen Mittelpunkt gehende Erzeugende der Fläche.

Hiernach erhält man folgende Construction der dreifach berührenden Ebene: vom Mittelpunkte $a=0, b=0, c=0$ des doppelt berührenden Kegels $b^{2}-4 a c=0$ lege man an den einfach berührenden Kegel $q^{2}-4 p r=0$ die beiden Tangentialebenen. Die eine derselben, $p t_{0}^{2}+q t_{0}+r=0$, ist beiden Kegeln gemeinschaftlich. Der anderen entspricht eine bestimmte Tangentialebene des Kegels $b^{2}-4 a c=0$, welche aus derselben eine durch den Punkt $a=0, b=0$, $c=0$ gehende Erzeugende ausschneidet. Die zweite durch diese Erzeugende gehende Tangentialebene des Kegels $b^{2}-4 a c=0$ ist die dreifach berührende Ebene der Fläche, welche aus derselben drei Erzeugende und einen einfachen Kegelschnitt ausschneidet.

Da im allgemeinen Falle der einfache Kegelschnitt mit der Doppelcurve Journal für $\overline{\text { Mathematik Bd.LXVII. Heft } 1 .}$ 
sechsten Grades drei Punkte gemein hat und derselbe im vorliegenden Falle mit dem Doppelkegelschnitt einen Punkt gemein hat, so hat derselbe mit der Doppelcurve vierten Grades zwei Punkte-gemein.

Ein Kegelschnitt, welcher mit einer Raumcurve vierten Grades mit zwei scheinbaren Doppelpunkten zwei Punkte gemein hat, bestimmt mit derselben im Allgemeinen eine geradlinige Fläche zehnten Grades.

Wir erhalten also den Satz:

Liegen auf einer geradlinigen Fläche füften Grades ein einfacher Kegelschnitt und eine Doppelcurve vierten Grades, so geht durch diese beiden Linien noch eine zweite geradlinige Fläche fünften Grades hindurch, von der jede Erzeugende den Kegelschnitt einmal und die Doppelcurve vierten Grades zweimal schneidet.

Zu den geradlinigen Flächen fünften Grades, welche einen doppelten Kegelschnitt und eine Doppelcurve vierten Grades besitzen, gehören auch die abwickelbaren Flächen fünften Grades.

[Die Doppelcurve der abwickelbaren Flächen muss jedesmal zerfallen in die Rückkehrkante und eine eigentliche Doppelcurve; die letztere fehlt nur bei den abwickelbaren Flächen vierten Grades. Da auf einer abwickelbaren Fläche überhaupt eine Leitgerade nicht vorhanden sein kann, so müssen die abwickelbaren Flächen fünften Grades sich unter denen befinden, bei welchen ein Kegelschnitt reducibler Theil der Doppelcurve ist.]

In Betreff der abwickelbaren Flächen fünfter Ordnung sei es gestattet, auf die schon genannte Arbeit des Verf. zu verweisen, wo auch die Literatur über dieselben möglichst vollständig angegeben ist.

Wir benutzen die abwickelbaren Flächen, um den letztgenannten Satz an einem Beispiele zu erläutern, und stellen zu diesem Zwecke vorher einige auf dieselben bezüglichen Gleichungen zusammen.

Gleichung der umhüllenden Ebene:

$$
a t^{4}+4 b t^{3}+6 c t^{2}+e=0 .
$$

Jede Erzeugende liegt ferner auf den Ebenen:

$$
\begin{aligned}
a t^{2}+3 b t+3 c & =0, \\
b t^{3}+3 c t^{2}+e & =0, \\
a t^{4}-6 c t^{2}-3 e & =0 .
\end{aligned}
$$

Die Rückkehrkante ist Durchschnitt der Kegel

$$
a e+3 c^{2}=0, \quad 3 b^{2}-4 a c=0 .
$$


Doppelter Kegelschnitt

$$
\begin{aligned}
& b=0, \quad a e-9 c^{2}=0 . \\
& e=0, \quad 3 a c-2 b^{2}=0 .
\end{aligned}
$$

Einfacher Kegelschnitt

Gleichung der abwickelbaren Fläche:

$$
a\left(a e+3 c^{2}\right)^{2}-6 c\left(a e+3 c^{2}\right)\left(3 b^{2}-4 a c\right)-3 e\left(3 b^{2}-4 a c\right)^{2}=0 .
$$

Durch einen Punkt des einfachen Kegelschnitts $a: b: c: e=6:-3 t: t^{2}: 0$ legen wir eine durch die Doppelcurve vierten Grades gehende Fläche zweiten Grades

$$
\left(a e+3 c^{2}\right)-t^{2}\left(3 b^{2}-4 a c\right)=0 .
$$

Die Tangentialebene derselben in dem betrachteten Punkte ist

$$
2 a t^{4}+9 b t^{3}+15 c t^{2}+3 e=0 .
$$

Dieselbe schneidet aus der Fläche zweiten Grades zwei Gerade aus, welche beziehlich in den Ebenen $a t^{2}+3 b t+3 c=0$ und $2 a t^{2}+3 b t-3 c=0$ liegen.

Jede dieser beiden Geraden schneidet den einfachen Kegelschnitt einmal und die Doppelcurve vierten Grades in zwei unendlich nahen oder getrennten Punkten. Die erste Gerade gehört der abwickelbaren Fläche an, die zweite aber erzeugt die geradlinige Fläche fünften Grades

$$
\begin{aligned}
2 a t^{4}+9 b t^{3}+15 c t^{2}+3 e & =0 \\
2 a t^{2}+3 b t-3 c & =0 \\
4 a t^{4}-24 c t^{2}-3 e & =0 \\
2 b t^{3}+6 c t^{2}+e & =0 .
\end{aligned}
$$

Die Ebene $b=0$ schneidet den doppelten Kegelschnitt der Fläche aus $b=0$, $a e+9 c^{2}=0$. Die Gleichung der geradlinigen Fläche ist

$$
4 a\left(a e+3 c^{2}\right)^{2}-24 c\left(a e+3 c^{2}\right)\left(3 b^{2}-4 a c\right)-3 e\left(3 b^{2}-4 a c\right)^{2}=0 .
$$

Die Doppelcurve vierten Grades der betrachteten Flächen kann nun selbst wieder in zwei Kegelschnitfe zerfallen, so dass dann die geradlinige Fläche fünften Grades drei doppelte Kegelschnitte besitzt.

Die Bedingung hierfür stellen wir folgendermassen auf.

Zwischen den sechs Ausdrücken $a, b, c, p, q, r$ besteht der Voraussetzung zufolge die eine identische Relation

$$
a t_{0}^{4}+b t_{0}^{2}+c=p t_{0}^{2}+q t_{0}+r ;
$$

weil es aber bloss vier homogene, von einander unabhängige Coordinaten giebt, so giebt es zwischen diesen sechs Ausdrücken noch eine solche identische Relation, welche wir gleich in die Form

setzen können.

$$
\alpha^{2} a-\gamma^{2} c=\lambda p+\mu q+\nu r
$$


Wir erhalten aus den Gleichungen der beiden Kegel, welche durch die Doppelcurve hindurchgehen,

$$
\begin{aligned}
& p^{2}-a\left(p t_{0}^{2}-q t_{0}+r\right)=0 \\
& r^{2}-c\left(p t_{0}^{2}-q t_{0}+r\right)=0
\end{aligned}
$$

diejenige des dritten durch die Doppelcurve gehenden Kegels zweiten Grades mit der Spitze $p=0, q=0, r=0$,

$$
\alpha^{2} p^{2}-\gamma^{2} r^{2}-(\lambda p+\mu q+\nu r)\left(p t_{0}^{2}-q t_{0}+r\right)=0 .
$$

Die Richtung der Tangenten der Curve im Doppelpunkt wird gegeben durch die Gleichungen

$$
\begin{aligned}
p t_{0}^{2}-q t_{0}+r & =0, & \alpha^{2} p^{2}-\gamma^{2} r^{2} & =0, \\
\alpha p+\gamma r & =0, & \alpha p-\gamma r & =0 .
\end{aligned}
$$

Wenn also die Curve vierten Grades in zwei Kegelschnitte zerfällt, mithin die Gleichung $\alpha^{2} p^{2}-\gamma^{2} r^{2}-(\lambda p+\mu q+\nu r)\left(p t_{0}^{2}-q t_{0}+r\right)=0$ das Product zweier linearen Factoren ist, so müssen dieselben die Form haben

$$
\begin{aligned}
& \alpha p-\gamma r+\sigma\left(p t_{0}^{2}-q t_{0}+r\right)=0, \\
& \alpha p+\gamma r+\sigma_{1}\left(p t_{0}^{2}-q t_{0}+r\right)=0 .
\end{aligned}
$$

Wenn wir die Producte identificiren, ergiebt sich

$$
\alpha\left(\sigma+\sigma_{1}\right) p+\gamma\left(\sigma-\sigma_{1}\right) r+\sigma \sigma_{1}\left(p t_{0}^{2}-q t_{0}+r\right)=-(\lambda p+\mu q+\nu r),
$$

$\sigma \sigma_{1} t_{0}=\mu, \quad \sigma_{1}=\frac{\mu}{\sigma t_{0}}, \quad \alpha\left(\sigma+\frac{\mu}{\sigma t_{0}}\right)+\lambda+\mu t_{0}=0, \quad \gamma\left(\sigma-\frac{\mu}{\sigma t_{0}}\right)+\nu+\frac{\mu}{t_{0}}=0$.

Finden diese Gleichungen statt, d. h. ist

$$
\alpha^{2} a-\gamma^{2} c=-\alpha\left(\sigma+\frac{\mu}{\sigma t_{0}}\right) p-\gamma\left(\sigma-\frac{\mu}{\sigma t_{0}}\right) r-\frac{\mu}{t_{0}}\left(p t_{0}^{2}-q t_{0}+r\right)
$$

für irgend welche Werthe von $\alpha, \gamma, \mu$, $\sigma$, so zerfällt die Doppelcurve vierten Grades in zwei Kegelschnitte, die zwei Punkte gemein haben.

Es giebt also geradlinige Flächen fünften Grades mit drei doppelten. Kegelschnitten. Diese drei Kegelschnitte haben einen Punkt gemein und schneiden sich zu je zweien in noch je einem Punkte. - (IX.)

Oder umgekehrt:

Legt man in die drei Seitenflächen $p, q, r$ eines Tetraeders pqrs je einen Kegelschnitt, der durch die Ecken der Seitenflächen desselben hindurchgeht, so sind diese drei Kegelschnitte im Allgemeinen die vollständige Doppelcurve einer geradlinigen Fläche fünften Grades.

Es ist auch möglich, dass die drei Punkte, in welchen sich die drei 
Kegelschnitte zu je zweien schneiden, in einen zusammenfallen, dass also die Ebenen der drei Kegelschnitte durch dieselbe Gerade gehen.

In diesem Punkte haben jedoch die drei Kegelschnitte nothwendig eine gemeinschaftliche Tangentialebene, während sie eine solche in dem anderen, ihnen gemeinsamen Punkte nicht haben dürfen, weil sonst die Fläche zweiten Grades, auf welcher die drei Kegelschnitte in diesem Falle liegen würden, ein reducibler Theil der geradlinigen Fläche fünften Grades sein würde.

Eine solche Fläche hat nun im Allgemeinen einen einfachen Leitkegelschnitt, welcher mit je zweien der Doppelkegelschnitte noch je eine zweite geradlinige Fläche fünften Grades bestimmt. -

Ein anderer Grund des Zerfallens der Doppelcurve sechsten Grades der allgemeinen geradlinigen Fläche fünften Grades und erster Klasse ist der, dass Doppelerzeugende auftreten.

In dem Falle, dass sich auf der Fläche eine gerade Leitlinie befindet, durch deren jeden Punkt nur eine Erzeugende geht, kann eine Doppelerzeugende nur auftreten, wenn sie mit der Geraden zusammenfällt.

Ist auf der Fläche ein einfacher Kegelschnitt vorhanden, so muss die Doppelerzeugende, wenn eine solche vorhanden, in der Ebene dieses Kegelschnitts liegen; mehr als eine solche kann es in diesem Falle nicht geben. - (X.)

Eine geradlinige Fläche fünften Grades kann auch eine dreifache Erzeugende haben.

Jede Ebene, welche durch eine dreifache Erzeugende einer geradlinigen Fläche fünften Grades geht, schneidet aus der Fläche noch ein Gebilde zweiten Grades aus. Da nun, wie oben bemerkt, auf einer geradlinigen Fläche fünften Grades höchstens ein einfacher Kegelschnitt liegen kann, so müssen diese Gebilde zweiten Grades in zwei Gerade zerfallen, und es ist daher die dreifache Gerade zugleich eine gerade Leitlinie für die Erzeugenden der Fläche; als solche kann dieselbe nur vom ersten Grade sein; da mit ihr die Erzeugende dreimal zusammenfällt, so ist diese Linie überhaupt eine vierfache Gerade der Fläche.

S. 3.

Besondere Betrachtung der zur zweiten Riemannschen Klasse gehörenden geradlinigen Flächen fünften Grades.

In der allgemeinen Betrachtung der geradlinigen Flächen fünften Grades ist gezeigt worden, dass die Flächen der zweiten Klasse stets eine unendliche Schaar von Curven dritten Grades ohne Doppelpunkte enthalten. 
Es ist also hier die Aufgabe zu lösen, zwei ebene Curven dritten Grades, - die nicht in derselben Ebene liegen, - in allgemeinster Weise reciprok so aufeinander zu beziehen, dass einem jeden Punkte der einen ein Punkt der anderen entspreche.

Damit dann durch die geraden Verbindungslinien entsprechender Punkte beider Curven eine geradlinige Fläche des fünften Grades bestimmt werde, ist erforderlich, dass ein Punkt der einen Curve zusammenfällt mit dem ihm entsprechenden Punkte der anderen Curve.

Wir lösen die genannte Aufgabe durch rein algebraische Betrachtungen.

Die beiden Curven dritten Grades seien in ihren Ebenen gegeben durch die Gleichungen

$$
\begin{array}{ll}
x: y: z=1: t: \sqrt{\psi_{1}(t)} ; & \psi_{1} t=4 t^{3}-g_{2} t-g_{3}, \\
u: v: w=1: s: \sqrt{\psi_{2}(s)} ; & \psi_{2} s=4 s^{3}-g_{2}^{\prime} s-g_{3}^{\prime} .
\end{array}
$$

Jedem Werthe von $t$ entsprechen zwei Punkte der ersten Curve; jedem Punkte der ersten Curve soll ein Punkt der zweiten Curve entsprechen, jedem Punkte der zweiten Curve entspricht ein Werth von $s$ : also entsprechen jedem Werthe von $t$ zwei Werthe von $s$. Ebenso wird gezeigt, dass jedem Werthe von $s$ zwei Werthe von $t$ entsprechen. Es besteht also zwischen $s$ und $t$ eine Gleichung, die in Bezug auf beide vom zweiten Grade ist:

$$
\begin{gathered}
f s^{2}+2 g s+h=f^{\prime} t^{2}+2 g^{\prime} t+h^{\prime}=0 . \\
s=\frac{-g \pm \sqrt{g^{2}-f h}}{f} ; \quad t=\frac{-g^{\prime} \pm \sqrt{g^{\prime 2}-f^{\prime} h^{\prime}}}{f^{\prime}} .
\end{gathered}
$$

Den Werthen von $t$, für welche $\psi_{1}(t)=0$ ist, sowie dem Werthe $t=\infty$ entspricht nur ein Punkt der Curve, also auch nur ein Werth von $s$; es darf sich daher auch nur ein Werth von $s$ aus dieser Gleichung ergeben; also ist die Discriminante $g^{2}-f h$ von $\psi_{1}(t)$ nur durch einen constanten Factor verschieden; dasselbe gilt für $g^{\prime 2}-f^{\prime} h^{\prime}$ und $\psi_{2}(s)$.

Bezeichnen wir den Werth von $s$, der dem Werthe $t=\infty$ entspricht, mit $s_{0}$, und mit $t_{0}$ den Werth von $t$, der dem Werthe $s=\infty$ entspricht, den Fall, dass die Werthe $s=\infty, t=\infty$ einander entsprechen, betrachten wir nachher gesondert -, so hat die Gleichung die Form

$\left(t-t_{0}\right)^{2}\left(s-s_{0}\right)^{2}+$ Glieder, die $s^{2}$ und $t^{2}$ nicht mehr enthalten:

$$
\begin{gathered}
\left(t-t_{0}\right)^{2}\left(s-s_{0_{1}}\right)^{2}-2 a\left(t-t_{0}\right)\left(s-s_{0}\right)-4 b\left(t-t_{0}\right)-4 c\left(s-s_{0}\right)+d=0, \\
{\left[\left(t-t_{0}\right)\left(s-s_{0}\right)-a\right]^{2}-4 b\left(t-t_{0}\right)-4 c\left(s-s_{0}\right)+d^{\prime}=0 .}
\end{gathered}
$$


Die Discriminanten sind

$$
\begin{aligned}
& 4 c\left(t-t_{0}\right)^{3}-d^{\prime}\left(t-t_{0}\right)^{2}+4 a b\left(t-t_{0}\right)+4 b^{2}, \\
& 4 b\left(s-s_{0}\right)-d^{\prime}\left(s-s_{0}\right)^{2}+4 a c\left(s-s_{0}\right)+4 c^{2} .
\end{aligned}
$$

Die Constante $b$ kann nicht gleich Null werden, ohne dass $c$ gleichzeitig Null wird; sonst wäre $s$ rational ausdrückbar durch $t$ und $\sqrt{4 c\left(t-t_{0}\right)-d^{\prime}}$ und nicht durch $t$ und $\sqrt{\psi t}$. Wir nehmen an, weder $b$ noch $c$ sei gleich Null; dann kann man durch die Substitution $t-t_{0}=\varkappa\left(t^{\prime}-t_{0}^{\prime}\right), s-s_{0}=\lambda\left(s^{\prime}-s_{0}^{\prime}\right)$ und zweckmässige Wahl von $\varkappa$ und $\lambda$ bewirken, dass in der neuen Gleichung $b^{\prime}=c^{\prime}$ wird; wir schreiben also, ohne der Allgemeinheit Abbruch zu thun

$$
\left[\left(t-t_{0}\right)\left(s-s_{0}\right)-a\right]^{2}-4 b\left(s-s_{0}\right)-4 b\left(t-t_{0}\right)+d b=0 .
$$

Die nothwendigen und hinreichenden Bedingungen dafür, dass die Discriminanten in reducirter Form auftreten, sind

Die Discriminanten sind dann

$$
d=-12 t_{0} ; \quad s_{0}=t_{0} .
$$

$$
\begin{aligned}
& b\left[4 t^{3}-\left(12 t_{0}^{2}-4 a\right) t-\left(-8 t_{0}^{3}+2 a t_{0}-4 b\right)\right], \\
& b\left[4 s^{3}-\left(12 t_{0}^{2}-4 a\right) s-\left(-8 t_{0}^{3}+2 a t_{0}-4 b\right)\right],
\end{aligned}
$$

also haben beide Curven dieselben Invarianten, und es ist $\left.\psi_{1}(t)=\psi_{2}(t)^{*}\right)$.

Hieraus folgt, dass die beiden Curven dritten Grades einander projectivisch sind, d. h. dass die eine als eine Centralprojection der anderen angesehen werden kann, weil die Gleichung der einen in Bezug auf die homogenen Coordinaten $x: y: z$

$$
z^{2} x=4 y^{3}-g_{2} y x^{2}-g_{3} x^{3}
$$

übereinstimmt mit der Gleichung der anderen

$$
w^{2} u=4 v^{3}-g_{2} v u^{2}-g_{3} u^{3}
$$

in Bezug auf die homogenen Coordinaten $u: v: w$.

Wir haben also den Satz:

Alle Curven dritten Grades, welche auf einer geradlinigen Fläche fünften Grades und zweiter Klasse liegen, können aus demselben Kegel dritten Grades ausgeschnitten werden.

Setzen wir

$$
b\left[4 t^{3}-\left(12 t_{0}^{2}-4 a\right) t-\left(-8 t_{0}^{3}+2 a t_{0}-4 b\right)\right]=b . \psi(t)=b\left(4 t^{3}-g_{2} t-g_{3}\right),
$$

so ergiebt sich

*) Dies würde sich auch aus den Sätzen des Herrn Aronhold (Monatsberichte der Acad. der Wissensch. 1861, pag. 462 u. f.) haben ableiten lassen. 


$$
\begin{gathered}
4 a=12 t_{0}^{2}-g_{2} ; \quad a=\frac{\psi^{\prime}\left(t_{0}\right)}{4}, \quad 4 b=\psi\left(t_{0}\right) ; \\
s-s_{0}=\frac{\psi\left(t_{0}\right)+\frac{1}{2} \psi^{\prime}\left(t_{0}\right)\left(t-t_{0}\right)+\sqrt{\psi\left(t_{0}\right)} \cdot \sqrt{\psi(t)}}{2\left(t-t_{0}\right)^{2}}, \\
s=\frac{\sqrt{\psi\left(t_{0}\right)} \cdot \sqrt{\psi(t)}+2 t_{0} t\left(t_{0}+t\right)-\frac{1}{2} g_{2}\left(t_{0}+t\right)-g_{3}}{2\left(t-t_{0}\right)^{2}}, \\
s=\frac{1}{4}\left(\frac{\sqrt{\psi\left(t_{0}\right)}+\sqrt{\psi(t)}}{t-t_{0}}\right)^{2}-\left(t_{0}+t\right) .
\end{gathered}
$$

Dem Werthe $t=t_{0}$ und $\sqrt{\psi(t)}=\sqrt{\psi\left(t_{0}\right)}$ entspricht $s=\infty$.

Diese Formeln sind Ausdrücke des Additionstheorems der elliptischen Function $\wp u=x$, welche durch die Differentialgleichung $\left(\frac{d x}{d u}\right)^{2}=4 x^{3}-g_{2} x-g_{3}$ und die Anfangsbedingung $\wp(0)=\infty$ definirt ist, und welche Herr Weierstrass in seinen Vorlesungen zu Grunde legt.

Durch die angegebenen Formeln ist nun auch $\sqrt{\psi(s)}$ bis auf das Vorzeichen bestimmt, d. h. durch $t$ und $\sqrt{\psi(t)}$ ausdrückbar; wir treffen nun die Bestimmung, indem wir nöthigenfalls $w$ mit $-w$ vertauschen, dass für den Punkt $t=\infty$, welchem $s=t_{0}$ entspricht, der zugehörende Werth von $\sqrt{\psi s}$ auch dem Zeichen nach mit $\sqrt{\psi t_{0}}$ übereinstimme. Wir setzen deswegen voraus, $\sqrt{\psi t_{0}}$ sei nicht gleich Null und dem Zeichen nach fixirt. Dann gilt die Formel

$$
\sqrt{\psi s}=\left(\frac{\psi(t)}{\left(t-t_{0}\right)^{3}}-\frac{1}{4} \frac{\psi^{\prime}(t)}{\left(t-t_{0}\right)^{2}}\right) \sqrt{\psi t_{0}}-\left(\frac{\psi\left(t_{0}\right)}{\left(t_{0}-t\right)^{3}}-\frac{1}{4} \frac{\psi^{\prime}\left(t_{0}\right)}{\left(t_{0}-t\right)^{2}}\right) \sqrt{\psi t}
$$

und man erhält die Ausdrücke von $t$ und $\sqrt{\psi t}$ durch $s$ und $\sqrt{\psi s}$ mittelst Buchstabenvertauschung.

Es ist ferner

und

$$
s+t_{0}+t=\frac{1}{4}\left\{\frac{\sqrt{\psi t_{0}}+\sqrt{\psi t}}{t-t_{0}}\right\}^{2}=\frac{1}{4}\left\{\frac{\sqrt{\psi s_{0}}+\sqrt{\psi s}}{s-s_{0}}\right\}^{2}
$$

entsprechend der Formel

$$
\frac{\sqrt{\psi t_{0}}+\sqrt{\psi t}}{t-t_{0}}=\frac{\sqrt{\psi s_{0}}+\sqrt{\psi s}}{s-s_{0}}
$$

$$
\frac{\wp^{\prime}(a-u)+\wp^{\prime} a}{\wp(a-u)-\wp a}=\frac{\wp^{\prime}(u)+\wp^{\prime} a}{\wp u-\wp a} .
$$

Dass die Zeichenbestimmung richtig gemacht ist, davon kann man sich auf folgende Weise überzeugen.

Aus den obigen Formeln ergiebt sich für Werthe von $t$, die $t_{0}$ benachbart sind, und für welche $\sqrt{\psi t}$ mit $\sqrt{\psi t_{0}}$ dem Zeichen nach übereinstimmt: 


$$
\begin{aligned}
s & =\frac{\psi t_{0}}{\left(t-t_{0}\right)^{2}}+\cdots, \\
\sqrt{\psi s} & =\frac{2 \cdot \psi t_{0} \cdot \sqrt{\psi t_{0}}}{\left(t-t_{0}\right)^{3}}+\cdots,
\end{aligned}
$$

und für unendlich grosse Werthe von $t$,

$$
\begin{aligned}
s & =s_{0}+\frac{\sqrt{\psi t_{0}} \cdot \sqrt{\psi t}}{2 t^{2}}+\cdots, \\
\sqrt{\psi s} & =\sqrt{\psi t_{0}}+\cdots
\end{aligned}
$$

Diese Werthepaare lassen eine andere Zeichenbestimmung nicht zu.

Wir denken uns nun die eine Curve projectivisch so geändert, dass dieselbe der anderen congruent wird und darauf mit derselben zur Deckung gebracht.

Es kann dies stets so geschehen, dass der Punkt $s, \sqrt{\psi s}$ zusammenfällt mit dem Punkte $t=s, \sqrt{\psi t}=\sqrt{\psi s}$.

Entspricht nun dem Punkte $t=t_{1}, \sqrt{\psi t}=\sqrt{\psi t_{1}}$, ein Punkt $s=t_{2}$, $\sqrt{\psi s}=\sqrt{\psi t_{2}}$, so sagen unsere Gleichungen aus, dass auch dem Punkte $s=t_{1}$, $\sqrt{\psi s}=\sqrt{\psi t_{1}}$ der Punkt $t=t_{2}, \sqrt{\psi t}=\sqrt{\psi t_{2}}$ entspreche. Es entspricht daher dem Punkte $t=t_{1}, \quad \sqrt{\psi t}=\sqrt{\bar{\psi} t_{1}}$ der Curve dritten Grades derselbe Punkt $t=t_{2}, \sqrt{\psi t}=\sqrt{\psi t_{2}}$, man möge ihn als der ersten oder als der zweiten Curve angehörig betrachten.

Wir bezeichnen den Punkt $1: t_{0}: \sqrt{\psi t_{0}}$ mit $x_{0}: y_{0}: z_{0}$, den Punkt $1: t: \sqrt{\psi t}$ mit $x_{1}: y_{1}: z_{1}$ und den entsprechenden Punkt $1: s: \sqrt{\psi s}$ mit $x_{2}: y_{2}: z_{2}$, so tritt an die Stelle der Gleichung

die folgende

$$
\frac{\sqrt{\psi t_{0}}+\sqrt{\psi t}}{t-t_{0}}=\frac{\sqrt{\psi s_{0}}+\sqrt{\psi s}}{s-s_{0}}
$$

$$
\frac{z_{1}+z_{0}}{y_{1}-y_{0}}=\frac{z_{2}+z_{0}}{y_{2}-y_{0}}
$$

welche aussagt, dass die drei Punkte

$$
x_{1}: y_{1}: z_{1} ; \quad x_{2}: y_{2}: z_{2} ; \quad x_{0}: y_{0}:-z_{0}
$$

auf einer geraden Linie liegen. Es geht also die Verbindungslinie je zweier entsprechenden Punkte der Curve stets durch denselben Punkt der Curve

$$
x: y: z=1: t_{0}:-\sqrt{\psi t_{0}} \text {. }
$$

Wir betrachten nun die Fälle, die wir oben ausgenommen hatten. Journal für Mathematik Bd. LXVII. Heft. 1. 
Entspricht dem Punkte $s=\infty$ entweder $t=\infty$ oder ein Werth, für den $\psi(t)=0$ ist, so besteht zwischen $s$ und $t$ eine Gleichung, welche in Bezug auf beide einzeln nur vom ersten Grade ist. Hierher gehört auch der Fall, dass in der zwischen $s$ und $t$ bestehenden Gleichung die Constanten $b$ und $c$ gleich Null werden, und diese Gleichung dadurch reducibel wird.

Diese Fälle kann man jedoch ohne Weiteres auf den behandelten dadurch zurückführen, dass man z. B. die Gleichung der Curve $s$ auf ein anderes Coordinatendreieck bezieht, welches eine andere Wendetangente als $u=0$ zu der einen Seite hat.

Dies ist auf achtfache Weise möglich, ohne dass sich die Gleichung der Curve ändert. Es entsprechen dann jedem Werthe von $s$ im Allgemeinen zwei Werthe von $t$, die zwischen ihnen bestehende Gleichung ist irreducibel, und dem Werthe $s=\infty$ entspricht ein Werth $t=t_{0}$, welcher nicht $\infty$ ist, und für welchen $\psi t$ nicht gleich Null ist.

[Entspricht dem Werthe $s=\infty, t=\infty$ oder eine Wurzel der Gleichung, welcher die Wendepunkte der Curve dritten Grades genügen,

$$
t^{4}-\frac{g_{2}}{2} t^{2}-g_{3} t-\frac{g_{2}^{2}}{48}=0
$$

welche zugleich die Drittheilung der ganzen Perioden der elliptischen Integrale ergiebt, die von den Invarianten $g_{2}$ und $g_{3}$ abhängen, - entspricht also einem Wendepunkte der einen Curve ein Wendepunkt der anderen, so werden die beiden Curven dritten Grades durch die entsprechenden Punkte einander projectivisch zugeordnet, und man kann diesen Fall stets durch eine geeignete Coordinatenumwandlung auf den Fall $s=t, \sqrt{\psi s}=\sqrt{\psi t}$ bringen.]

Wir erhalten nun folgende allgemeine Construction für die geradlinigen Flächen fünften Grades und zweiter Klasse:

Auf einer ebenen Curve dritten Grades nehme man einen Punkt fest an; so wird jedem Punkte der Curve ein anderer zugeordnet, der auf der Curve und mit dem betrachteten und dem festen Punkte in gerader Linie liegt. Man denke sich nun die Curve dritten Grades doppelt, so wird jedem Punkte der einen Curve ein nicht mit ihm zusammenfallender Punkt der anderen Curve eindeutig zugeordnet. Hierauf denke man sich die Ebenen beider Curven getrennt und die eine Curve in ihrer Ebene collinear verwandelt.

Lässt man sodann einen Punkt der einen Curve mit seinem entsprechenden Punkte auf der anderen Curve zusammenfallen, so ist der geometrische 
Ort der Verbindungslinien entsprechender Punkte beider Curven im Allgemeinen eine geradlinige Fläche fünften Grades und zweiter Klasse.

Die Ebene einer Curve dritten Grades enthält bei der erwähnten Construction ausser dieser Curve noch zwei Erzeugende der Fläche; der ebene Schnitt enthält also überhaupt $3+3+1=7$ Doppelpunkte. Davon gehen ab zwei Berührungspunkte der Ebene mit der Fläche, also bleiben fünf Doppelpunkte, welche der Fläche angehören.

Die Doppelcurve der betrachteten Flächen ist vom füften Grade. - (I.)

Durch jeden Punkt der Doppelcurve gehen zwei Erzeugende der Fläche, deren jede die Doppelcurve noch in zwei Punkten schneidet. Der Kegel, welcher die Doppelcurve zur Leitlinie und einen Punkt derselben zum Mittelpunkt hat, hat demnach zwei Doppelkanten, und da derselbe vom vierten Grade ist, so gehört die Curve im allgemeinen Falle in die Klasse $\varrho=1$.

Die reciproke Fläche der betrachteten ist von derselben Natur und hat also auch eine Doppelcurve fünften Grades.

Wir folgern hieraus, zu der ursprünglichen Fläche zurückkehrend, dass durch jeden Punkt des Raumes fünf Ebenen gehen, welche aus der Fläche zwei Erzeugende ausschneiden. Für jeden Punkt der Fläche haben drei dieser Ebenen die durch diesen Punkt gehende Erzeugende gemein; die zwei übrigen schneiden aus der Fläche zwei durch diesen Punkt gehende Curven dritten Grades aus.

Wir erhalten also den Satz:

Im allgemeinen Falle gehen durch jeden Punkt der betrachteten Fläche zwei ebene Curven dritten Grades hindurch.

Wenn die Doppelcurve fünften Grades zerfällt, so ist nothwendig unter den Theilen eine doppelte oder eine dreifache Gerade; denn sie kann nicht zerfallen in einen doppelten Kegelschnitt und eine Raumcurve dritten Grades; der Kegelschnitt würde eine einfache Linie der entstehenden Fläche werden.

Enthält die geradlinige Fläche fünften Grades eine dreifache gerade Leitlinie, so enthält sie ausser derselben im Allgemeinen einen doppelten Kegelschnitt, welcher die dreifache Gerade in einem Punkte schneidet. Und jede solche Fläche, deren allgemeine Gleichung

$$
(a p-b q)_{i}^{2} p^{\prime}+(a p-b q) s \cdot p^{\prime \prime} q^{\prime \prime}+s^{2} \cdot q^{\prime} \cdot p^{\prime \prime \prime} q^{\prime \prime \prime}=0
$$

ist, worin $p, q, p^{\prime}, q^{\prime}, p^{\prime \prime}, q^{\prime \prime}, p^{\prime \prime \prime}, q^{\prime \prime \prime}$ durch dieselbe Gerade gehen, ist eine geradlinige Fläche. - (II.)

Der doppelte Kegelschnitt $s=0, a p-b q=0$ kann auf dieselbe Weise, 
wie im vorigen Paragraphen erörtert, in zwei Gerade zerfallen; die eine davon wird eine doppelte Leitgerade, die andere eine Doppelerzeugende der Fläche. - (III.)

Auch kann die doppelte Leitgerade der dreifachen Leitgeraden unendlich nahe rücken. Es unterscheidet sich die dann entstehende Fläche bloss dadurch von der analogen im vorigen Paragraphen betrachteten, dass sie eine Doppelerzeugende weniger besitzt.

Enthält die geradlinige Fläche fünften Grades eine doppelte gerade Leitlinie, so enthält sie ausser derselben im Allgemeinen eine Doppelcurve vierten Grades mit zwei scheinbaren Doppelpunkten, welche von der einfachen Geraden in einem Punkte geschnitten wird. - (IV.)

Umgekehrt ist auch jede Fläche fünften Grades mit einer Doppelcurve vierten Grades und einer dieselbe in einem Punkte schneidenden doppelten Geraden eine geradlinige, denn jede durch die Doppelgerade gelegte Ebene schneidet noch eine Curve dritten Grades mit drei Doppelpunkten aus, welche also in drei Gerade zerfallen muss. -

Jedes durch die Doppelcurve vierten Grades gehende Hyperboloid schneidet aus der Fläche zwei Erzeugende aus, welche sich auf der Doppelgeraden schneiden.

Alle Ebenen, welche durch je zwei solche Erzeugende gelegt sind, die Tangentialebenen der Hyperboloide, umhüllen einen Kegel zweiten Grades. Die reciproke geradlinige Fläche dieser Fläche ist also die vorhin betrachtete II. mit einem doppelten Kegelschnitt.

Hat eine Fläche der zweiten Klasse eine doppelte Erzeugende, so schneidet eine durch dieselbe gelegte Ebene im Allgemeinen eine irreducible Curve dritten Grades aus. Der Schnitt einer solchen Ebene enthält demnach im Allgemeinen keinen Doppelpunkt, der nicht auf der Doppelerzeugenden läge.

Hieraus folgt, dass die Theile der Doppelcurve nur ebene Curven sein können, deren Ebene durch die Doppelgerade hindurchgeht. Es sind diese, wie sich bei näherer Betrachtung ergiebt, eine dreifache und eine zweifache Gerade. - (III.)

In diesem Falle werden alle Curven dritten Grades durch die Erzeugenden der Fläche projectivisch.

Es entsteht nun die Frage, welche Doppelcurve hat überhaupt eine geradlinige Fläche fünfter Ordnung, wenn die beiden Curven dritten Grades, die zu ihrer Construction dienen, durch die entsprechenden Punkte projectivisch sind? 
Wir legen durch den gemeinschaftlichen Punkt in der Ebene der einen Curve eine Gerade, in der Ebene der anderen Curve die entsprechende Gerade und durch beide Gerade eine Ebene. In dieser Ebene liegen zwei Erzeugende der Fläche. Nach einem bekannten Satze:

„Haben zwei in verschiedenen Ebenen liegende projectivische ebene

Strahlbüschel den Mittelpunkt mit einander gemein, so umhüllen alle Ebenen, welche durch je zwei entsprechende Strahlen beider Büschel gelegt werden, im Allgemeinen einen Kegel zweiten Grades, “ folgern wir, dass die definirten Ebenen, von denen jede zwei Erzeugende aus der Fläche ausschneidet, einen Kegel zweiten Grades umhüllen.

Die reciproke Fläche der eben betrachteten hat daher einen doppelten Kegelschnitt und ausserdem eine dreifache Gerade.

Wir schliessen also mit Bezug auf die obigen Angaben, dass unsere Fläche im Allgemeinen eine zweifache Leitgerade und eine Doppelcurve vierten Grades besitzt, also mit IV. identisch ist.

\section{\$. 4.}

Besondere Betrachtung der zur dritten Riemannschen Klasse gehörenden geradlinigen Flächen fünften Grades.

Bei der allgemeinen Untersuchung der verschiedenen geradlinigen Flächen fünften Grades haben sich zwei Fälle ergeben, in denen die Flächen zur dritten Klasse gehören. Der erste Fall ist der, dass die Fläche eine dreifache und eine zweifache gerade Leitlinie besitzt und der zweite der, dass die Fläche eine dreifache gerade Leitlinie von der Beschaffenheit besitzt, dass jede durch dieselbe hindurchgelegte Ebene aus der Fläche zwei Erzeugende ausschneidet, welche sich auf der dreifachen Geraden schneiden.

Wir werden zeigen, dass der zweite Fall als ein specieller Fall des ersten angesehen werden kann und beginnen mit der Betrachtung des ersten Falles.

Bezeichnet $p \lambda+q=0$ eine beliebige durch die dreifache Gerade gehende Ebene, $r \dot{\mu}+s=0$ eine beliebige Ebene durch die zweifache Leitgerade, so entsprechen jedem. Werthe von $\lambda$ zwei Werthe von $\mu$ und jedem Werthe von $\mu$ drei Werthe von $\lambda$. Denn jede Ebene $p \lambda+q=0$ schneidet aus der Fläche zwei sich auf der Geraden $r=0, s=0$ schneidende Erzeugende aus, und jede Ebene $r \mu+s=0$ schneidet drei Erzeugende aus, die sich auf der Geraden $p=0, q=0$ schneiden. Es besteht also zwischen $\lambda$ und $\mu$ eine 
algebraische Gleichung $f(\lambda, \mu)=0$, welche in Bezug auf $\lambda$ vom dritten und in Bezug auf $\mu$ vom zweiten Grade ist.

Aus dieser Gleichung erhält man die Gleichung der Fläche, wenn man $-\frac{q}{p}$ für $\lambda$ und $-\frac{s}{r}$ für $\mu$ setzt und darauf mit $p^{3} r^{2}$ multiplicirt. -

Legt man durch eine Erzeugende eine Ebene, so schneidet diese im Allgemeinen aus der Fläche eine Curve vierten Grades mit einem Doppelpunkte aus.

Mit Bezug auf eine solche Curve ergeben sich nun folgende Sätze.

Fasst man in einer ebenen Curve vierten Grades mit einem Doppelpunkte diesen Doppelpunkt und einen Punt der Curve als Mittelpunkte zweier geradlinigen ebenen Strahlbüschel auf, so werden die Strahlen derselben durch die Punkte der Curve einander zugeordnet.

Macht man mit diesen Strahlbüscheln zwei $\left\{\begin{array}{c}\text { Ebenenbüschel } \\ \text { Gerade }\end{array}\right\}$ projectivisch, so ist der Ort der $\left\{\begin{array}{c}\text { Durchschnittslinien } \\ \text { Verbindungsgeraden }\end{array}\right\}$ entsprechender Elemente eine geradlinige Fläche fünften Grades von der dritten Riemannschen Klasse.

Man erhält ferner den Satz:

Macht man eine Gerade projectivisch mit dem Strahlbiischel des Doppelpunkts und lässt einen Punkt derselben mit einem der beiden ihm auf der Curve entsprechenden Punkte zusammenfallen, so ist der geometrische Ort der Verbindungslinien entsprechender Punkte eine geradlinige Fläche füften Grades oon der betrachteten Art.

Ein analoger Satz gilt für eine Gerade, welche dem anderen Strahlbüschel projectivisch ist und durch den Doppelpunkt der Curve vierten Grades gelegt wird.

Beiläufig sei noch folgender Satz erwähnt:

Jedes Hyperboloid, welches durch drei Erzeugende der Fläche geht, von denen nicht zwei einander schneiden, schneidet aus der Fläche noch zwei Erzeugende aus.

Das Hyperboloid enthält nämlich die beiden Leitgeraden, hat also schon eine Curve vom Grade $3+2+3=8$ mit der Fläche gemein. Da nun auf dieser ein Kegelschnitt nicht vorhanden ist, so muss es noch zwei Erzeu-. gende aus der Fläche ausschneiden.

Wir gehen nun über zur Betrachtung des zweiten Falles. 
Durch eine Erzeugende legen wir eine Ebene, welche eine Curve vierten Grades mit einem Doppelpunkte ausschneidet. Die Gleichung dieser Curve sei

$$
p q r^{2}+2 u_{3} r+u_{4}=0, \quad s=0
$$

$p=0, q=0$ sind die Tangenten im Doppelpunkte; wir können annehmen, dass die beiden Ebenen $p=0$ und $q=0$ durch die dreifache Leitgerade hindurch gelegt seien; $u_{3}$ und $u_{4}$ sind homogene Functionen dritten und vierten Grades von $p$ und $q$.

Jedem Punkte der dreifachen Geraden entspricht eine durch dieselbe gehende Ebene, welche die beiden Erzeugenden enthält, die von dem Punkte ausgehen, also auch in der Ebene der Curve eine Gerade durch den Doppelpunkt; mithin entsprechen jedem Punkte der dreifachen Geraden zwei Punkte der Curve.

Damit wirklich die so bestimmte Fläche nur vom fünften Grade werde, darf dem Punkte, in welchem die dreifache Gerade die Ebene schneidet, nur ein Punkt der Curve in endlich grosser Entfernung entsprechen, sonst würden zwei Erzeugende in die Ebene eintreten; also muss der andere entsprechende Punkt der Curve unendlich nahe liegen. Hieraus folgt, dass die Erzeugende in der Ebene der Curve vierten Grades eine Tangente der Curve im Doppelpunkte sein muss.

Es sei $q=0, s=0$ die in der Ebene $s=0$ liegende Erzeugende. Nun entspricht jeder Ebene $p \lambda+q=0$ ein bestimmter Punkt der dreifachen Leitgeraden; derjenige nämlich, in welchem sich die beiden von dieser Ebene ausgeschnittenen Erzeugenden schneiden. Bestimmt man diesen Punkt durch den Werth der Constante $\mu$ in der Gleichung der durch den Punkt gelegten Ebene $r \mu+s=0$, so entspricht jedem $\mu$ ein $\lambda$ und jedem $\lambda$ ein $\mu$. Man kann nun die Ebene $r=0$ so wählen, dass sie durch den Punkt der dreifachen Geraden hindurchgeht, der der Ebene $p=0$ entspricht, ihr Schnitt mit der Ebene $s=0$ aber unverändert bleibt; dann können wir unbeschadet der Allgemeinheit dem Punkte $p=0, q=0, r \lambda+s=0$ die Ebene $p \lambda+q=0$ entsprechen lassen.

Es ist also die dreifache Gerade projectivisch dem Strahlbüschel des Doppelpunktes, und es besteht nur der Unterschied von der allgemeinen Construction, dass ein Punkt der Geraden, der einem unendlich nahen Punkte des Doppelpunkts entspricht, mit dem Doppelpunkte zur Coincidenz gebracht wird. 
Es handelt sich nun darum, die Gleichung der geradlinigen Fläche aufzustellen.

Setzen wir in der Gleichung der Curve vierten Grades $p \lambda+q=0$, so ergiebt sich

$$
-\lambda r^{2}+2 v_{3} r p+v_{4} p^{2}=0
$$

worin mit $v_{3}$ und $v_{4}$ die aus $u_{3}$ und $u_{4}$ durch die Substitution $q=-p \lambda$ hervorgehenden ganzen Functionen dritten und vierten Grades von $\lambda$ bezeichnet sind. Hieraus ergiebt sich

$$
\frac{p}{r}=\frac{-v_{3} \pm \sqrt{v_{3}^{2}+v_{4} \lambda}}{v_{4}}=\frac{-\lambda}{-v_{3} \mp \sqrt{v_{3}^{2}+v_{4} \lambda}} .
$$

Wir bestimmen nun ausser der Ebene $p \lambda+q=0$ eine zweite Ebene

$$
\alpha p+\beta q+\gamma r+\delta s=0,
$$

welche durch den Punkt $p=0, q=0, r \lambda+s=0$ und durch den Punkt

$$
p \lambda+q=0, \quad p+\frac{r \lambda}{-v_{3} \mp \sqrt{v_{3}^{2}+v_{4} \lambda}}=0, \quad s=0
$$

hindurchgeht, also die Ebene $p \lambda+q=0$ in einer Erzeugenden schneidet.

Eine solche Ebene ist

$$
\left(-v_{3} \mp \sqrt{v_{3}^{2}+v_{4} \lambda}\right) p+r \lambda+s=0 .
$$

Diese Ebene bestimmt also für jeden Werth von $\lambda$ mit der Ebene $p \lambda+q=0$ eine Erzeugende. Gehen wir zu der rationalen Form

$$
-p^{2} v_{4} \lambda-2(r \lambda+s) p v_{3}+(r \lambda+s)^{2}=0
$$

über, so erhalten wir als Gleichung der Fläche, indem wir $\lambda$ eliminiren

$$
U_{4} q-2(p s-r q) \cdot U_{3}+(p s-r q)^{2} p=0
$$

wo $U_{4}$ und $U_{3}$ die oben mit $u_{4}$ und $u_{3}$ bezeichneten homogenen Functionen von $p$ und $q$ sind.

[Wenn die Curve vierten Grades eine Spitze an Stelle des Doppelpunktes hat, ist die allgemeine Gleichung der Fläche

$$
U_{4} q-2(p s-r q) \cdot U_{3}+(p s-r q)^{2} q=0 \text {.] }
$$

Wendet man auf diese Fläche die Betrachtungen an, die im $\$$. 2 auf einen speciellen Fall derselben angewandt worden sind, so ergiebt sich, dass die Fläche sich längs der Geraden $p=0, q=0$ des Hyperboloids $p s-r q=0$ selbst berührt.

Diese Selbstberührung entspricht einer der dreifachen Leitlinie auf dem Hyperboloid überall unendlich nahe gerückten doppelten geraden Leitlinie. Jede Erzeugende der Fläche berührt nämlich das Hyperboloid und geht daher durch zwei unendlich nahe Erzeugende desselben. 
Der besseren Uebersicht wegen folgt hier eine Zusammenstellung der einzelnen Arten der geradlinigen Flächen fünften Grades, die wir in Bezug auf die Doppelcurve unterschieden haben.

A. Geradlinige Flächen fünften Grades und erster algebraischer Klasse. $(\varrho=0)$.

Die Doppelcurve ist:

1. teine vierfache Gerade,

II. eine Raumcurve sechsten Grades mit einem dreifachen Punkte,

III. eine dreifache Leitgerade und eine Raumcurve dritten Grades,

IV. eine dreifache Leitgerade, ein Kegelschnitt und eine Doppelerzeugende,

V.+ eine dreifache und eine zweifache Leitgerade nebst zwei Doppelerzeugenden; specieller Fall: beide Leitgerade sind unendlich nahe gerückt;

VI. eine zweifache Leitgerade und eine Raumcurve fünften Grades mit einem dreifachen Punkte,

VII. eine zweifache Leitgerade, eine Raumcurve vierten Grades mit einem Doppelpunkt und eine Doppelerzeugende,

VIII. ein Kegelschnitt und eine Raumcurve vierten Grades mit einem Doppelpunkt,

IX. Verein dreier Kegelschnitte,

X. eine Doppelerzeugende und eine Raumcurve fünften Grades mit einem zweifachen Punkte.

$B$. Geradlinige Flächen fünften Grades und zweiter algebraischer Klasse. $(\varrho=1)$.

Die Doppelcurve ist

I. eine Raumcurve fünften Grades,

II. eine dreifache gerade Leitlinie und ein doppelter Kegelschnitt,

III. + eine dreifache und eine zweifache gerade Leitlinie und eine Doppelerzeugende,

IV. eine zweifache gerade Leitlinie und eine Raumcurve vierten Grades.

C. Geradlinige Flächen fünften Grades und dritter algebraischer Klasse. $(\varphi=2)$.

+ Die Doppelcurve wird gebildet durch eine dreifache und eine zweifache gerade Leitlinie, welche einander auch unendlich nahe rücken können.

Berlin, im October 1866. 